\title{
Modal dynamics of structures with bladed isotropic rotors and its complexity for two-bladed rotors
}

\author{
Morten Hartvig Hansen \\ Dept. of Wind Energy, Technical University of Denmark, Frederiksborgvej 399, 4000 Roskilde, Denmark \\ Correspondence to: Morten H. Hansen (mhha@dtu.dk)
}

Received: 2 August 2016 - Published in Wind Energ. Sci. Discuss.: 23 August 2016

Accepted: 7 November 2016 - Published: 30 November 2016

\begin{abstract}
The modal dynamics of structures with bladed isotropic rotors is analyzed using Hill's method. First, analytical derivation of the periodic system matrix shows that isotropic rotors with more than two blades can be represented by an exact Fourier series with 3/rev (three per rotor revolution) as the highest order. For two-bladed rotors, the inverse mass matrix has an infinite Fourier series with harmonic components of decreasing norm; thus, the system matrix can be approximated by a truncated Fourier series of predictable accuracy. Second, a novel method for automatically identifying the principal solutions of Hill's eigenvalue problem is introduced. The corresponding periodic eigenvectors can be used to compute symmetric and antisymmetric components of the two-bladed rotor motion, as well as the additional forward and backward whirling components for rotors with more than two blades. To illustrate the use of these generic methods, a simple wind turbine model is set up with three degrees of freedom for each blade and seven degrees of freedom for the nacelle and drivetrain. First, the model parameters are tuned such that the low-order modal dynamics of a three-bladed $10 \mathrm{MW}$ turbine from previous studies is recaptured. Second, one blade is removed, leading to larger and higher harmonic terms in the system matrix. These harmonic terms lead to modal couplings for the two-bladed turbine that do not exist for the three-bladed turbine. A single mode of a two-bladed turbine will also have several resonance frequencies in both the ground-fixed and rotating frames of reference, which complicates the interpretation of simulated or measured turbine responses.
\end{abstract}

\section{Introduction}

A fundamental understanding of the modal dynamics of structures with bladed rotors is relevant for the design and analysis of wind turbines, helicopters, and other rotating machinery because their vibrational responses are composed of their structural modes. It is important to understand how these modes are excited by resonances or aeroelastic instabilities, i.e., at which frequencies and where on the structure or rotor the individual modes can be excited. Such knowledge is necessary not only for the interpretation of design simulations but also for the understanding of real measurements.

The modal dynamics of three-bladed turbines is well understood, also including the interaction with aerodynamic forces and a controller. For isotropic rotors, the Coleman transformation (Coleman and Feingold, 1958) is often used to transform the periodic system into a time-invariant sys- tem and then solving the associated eigenvalue problem with the blade motion described by the multi-blade coordinates (Hansen, 2003, 2004, 2007; van Engelen and Braam, 2004; Riziotis et al., 2008; Bir, 2008; Skjoldan and Hansen, 2009; Bergami and Hansen, 2016). The turbine modes may either be dominated by vibrations of the rotor support structure and drivetrain, e.g., tower bending and shaft torsion modes, or by blade vibrations which herein are called the rotor modes. The rotor modes of the three-bladed isotropic rotor consist of a symmetric mode and two whirling modes for each blade mode (edgewise/flapwise bending and torsion). In the whirling modes, the order of blade vibration describes a backward (regressive) and a forward (progressive) whirling direction relative to the rotor rotation. Due to the anisotropy of the rotor support, the rotor modes are not "pure", meaning that, for example, a backward whirling mode will also con- 
tain symmetric and forward whirling components when observed from the rotating blade frame of reference. The modal frequency obtained from the eigenvalue problem describes the frequency observed from the ground-fixed frame of reference in which the multi-blade coordinates describes the rotor motion. In the rotating blade frame, the symmetric rotor response will be observable at the same frequency but the backward and forward whirling components of a rotor mode will be shifted by $+1 /$ rev and $-1 /$ rev, respectively. Since a rotor mode is not pure, its response may therefore be observable at three different frequencies in a signal measured on the blade (Hansen, 2003, 2007).

For anisotropic three-bladed rotors, Floquet theory or Hill's method is needed to obtain an eigenvalue problem which leads to the periodic eigenvectors of the principal eigenvalue solutions (Skjoldan and Hansen, 2009; Skjoldan, 2009; Skjoldan and Hansen, 2009; Bottasso and Cacciola, 2015). To handle the frequency indeterminacy of the periodic eigenvalue solutions from these methods, Skjoldan and Hansen (2009) suggest to select the principal solution such that the harmonic components on the ground-fixed degrees of freedom are minimized. Bottasso and Cacciola (2015) introduce the concept of modal participation factors in which the norm of the individual harmonic components of a periodic eigenvector determines how much the particular component contributes to the response of the particular mode. They also introduce the concept of periodic Campbell diagrams to plot the frequencies of these harmonic components along with the principal frequency. All studies show that periodic mode shapes of turbines with three-bladed anisotropic rotors can contain harmonic components whose frequencies can be shifted more than $\pm 1 /$ rev from the principal modal frequency. The sizes of these higher harmonic components depend on the size of rotor anisotropy.

The modal analysis of structures with two-bladed rotors is complicated by the strong periodicity of the system. The use of Floquet theory or Hill's method is unavoidable, unless both rotor and support structure are isotropic with respect to rotation (Coleman and Feingold, 1958). Early studies (Warmbrodt and Friedmann, 1980; Wendell, 1982; Kirchgäßner, 1984) have used Floquet theory to investigate the aeroelastic stability of two-bladed rotors without focusing on their modal dynamics. However, Kirchgäßner (1984) introduces the concept of dominant eigenfrequencies for the harmonic components of the Floquet solutions with the largest magnitude in the corresponding eigenvector, and he plotted the frequencies of all harmonic components with magnitudes larger than a threshold relative to the dominant component in a periodic Campbell diagram similar to Bottasso and Cacciola (2015). A later study by Stol et al. (2002) considers the dynamic stability of a teetered two-bladed turbine using Floquet theory on a model with up to seven degrees of freedom. Their focus is mainly on the parametric excitation of the system and less on its modal dynamics. Recent studies of two-bladed turbines have focused on their aero- servo-elastic control (Solingen, 2015; Solingen et al., 2016a, b; Wang and Wright, 2016) and on their design loads (Kim et al., 2015). In the latter study, Kim et al. (2015) plots the spectrogram of the tower top signal obtained from nonlinear time simulations of a two-bladed turbine and compares it to the spectrogram of a three-bladed version of the same turbine. There are similarities between these spectrograms which lead the authors to conclude that two-bladed turbines have similar modes as three-bladed turbines. In experimental study of a scaled turbine, Larsen and Kim (2015) conclude that asymmetric rotor modes split into backward and forward whirling modes with $\pm 1 / \mathrm{rev}$, similar to whirling modes of three-bladed turbines except that there are also components at multiple of the rotor speed.

In this paper, the modal dynamics of structures with a rotor that have two or more blades are considered, first from a generic model-independent perspective and then with focus on the differences between the modal dynamics of two- and three-bladed turbines. In Sect. 2, analytical derivations of the linear equations of motion in a generic form and analytical inversion of the mass matrix show that the periodic system matrix for isotropic rotors with more than two blades has a finite and exact Fourier series, with 3/rev being the highest harmonic order. The system matrix for structures with two-bladed rotors has an infinite Fourier series of harmonic components that decrease in norm for increasing order. Using Hill's method to obtain the periodic mode shapes of the principal eigen-solutions, it is shown in Sect. 3 how the modal amplitudes for rotating blade degrees of freedom in the periodic eigenvectors can be used to decompose the rotor motion into symmetric and antisymmetric components for twobladed turbines and additional whirling components for rotors with more than two blades; we note, however, that antisymmetric components do not exist for odd numbers of blades and that whirling components do not exist for twobladed turbines. In Sect. 4, a low-fidelity kinematic model of a $10 \mathrm{MW}$ turbine consisting of three blade modes and seven degrees of freedom for the nacelle and drivetrain is used to exemplify the differences between the modal dynamics of two- and three-bladed turbines using the presented generic methods. It is shown that although a two-bladed turbine does not have whirling modes, the response of an antisymmetric rotor mode observed from a ground-fixed signal, such as a tower acceleration or moment, looks similar to the $\pm 1 /$ rev frequency splitting from the a whirling mode pair for a threebladed turbine. This similarity explains the incorrect conclusions made in the previous studies (Kim et al., 2015; Larsen and Kim, 2015). The present analytical study also shows that the additional harmonics observed for two-bladed rotors lead to several significant modal couplings when the frequencies of higher harmonic components in a periodic mode shape coincide with other modal frequencies. 


\section{Analytical system matrix for isotropic rotors}

Analytical expressions for the harmonic components of the periodic system matrix for structures with an isotropic rotor are derived in this section. The first-order state-space equation for a periodic system with the period $T$ is given by

$\dot{\boldsymbol{x}}=\mathbf{A}(t) \boldsymbol{x}$,

where $(\dot{)})=\mathrm{d} / \mathrm{d} t$ and $\mathbf{A}(t)=\mathbf{A}(t+T)$ is the $T$-periodic system matrix of dimension $2 N_{\mathrm{D}} \times 2 N_{\mathrm{D}}$, where $N_{\mathrm{D}}$ is the number of degrees of freedom (DOFs). With the state vector ordered as $\boldsymbol{x}=\{\boldsymbol{u}, \dot{\boldsymbol{u}}\}^{T}$ where $\boldsymbol{u}$ and $\dot{\boldsymbol{u}}$ are the DOFs and their time derivatives, the system matrix can be derived from linear second-order equations of motion as

$\mathbf{A}(t)=\left[\begin{array}{cc}\mathbf{0} & \mathbf{I} \\ -\mathbf{M}^{-1}(t) \mathbf{K}(t) & -\mathbf{M}^{-1}(t) \mathbf{C}(t)\end{array}\right]$,

where $\mathbf{I}$ is a identity matrix and $\mathbf{M}, \mathbf{C}$, and $\mathbf{K}$ are the $T$ periodic mass, gyroscopic/damping, and stiffness $N_{\mathrm{D}} \times N_{\mathrm{D}}$ matrices, respectively. Periodicity of these matrices ensures that the system matrix can be written as a Fourier series:

$\mathbf{A}(t)=\sum_{n=-\infty}^{\infty} \mathbf{A}_{n} e^{i n \Omega t}$,

where $\iota=\sqrt{-1}$ and $\Omega \equiv 2 \pi / T$ is the constant mean rotational speed. Note that the mean component of the system matrix $\mathbf{A}_{0}$ is a real matrix, and the complex matrices of the harmonic components come in conjugated pairs:

$\mathbf{A}_{-n}=\overline{\mathbf{A}}_{n}, \quad n=1,2,3, \ldots$,

where the bar denotes the complex conjugated operator. The periodic matrices of the second-order equations of motion are derived in the next section. In Sect. 2.2, the mass matrix is inverted analytically to obtain its Fourier series. The mean and harmonic component matrices of the periodic system matrix are finally presented in Sect. 2.3.

\subsection{Equations of motion}

Let the Lagrangian for a structure with a rotor be written as

$L=T(t, \boldsymbol{u}, \dot{\boldsymbol{u}})-V(\boldsymbol{u})$,

where it is assumed that the potential energy of the conservative forces is time-independent and only depends on the generalized coordinates $V=V(\boldsymbol{u})$, e.g., the elastic forces. The total kinetic energy is given by an integral of the kinetic energy of each particle over the entire volume $\mathcal{V}$ of the structure as

$T=\int_{\mathcal{V}} \frac{1}{2} \rho \dot{\boldsymbol{r}}^{T} \dot{\boldsymbol{r}} d \mathcal{V}$, where $(\cdot)^{T}$ denotes to the transpose matrix operator and $\dot{\boldsymbol{r}}$ is the velocity vector of the particle given as the time derivative of its position vector $\boldsymbol{r}=\boldsymbol{r}(t, \boldsymbol{u})$, which for the rotor part of the structure will be explicitly time-dependent.

Through substitution of the Lagrangian Eq. (5) with Eq. (6) into Lagrange's equations and linearization about a steady-state deflection of the structure $\boldsymbol{u}=\boldsymbol{u}_{0}$ and $\dot{\boldsymbol{u}}=\mathbf{0}$, the coefficients of the matrices of Eq. (2) can be written as (Meirovitch, 1970)

$$
\begin{aligned}
m_{i j} & =\int_{\mathcal{V}} \rho\left(\frac{\partial \boldsymbol{r}^{T}}{\partial u_{i}} \frac{\partial \boldsymbol{r}}{\partial u_{j}}\right) d \mathcal{V} \\
c_{i j} & =\frac{\partial}{\partial t} m_{i j}+\int_{\mathcal{V}} \rho\left(\frac{\partial \boldsymbol{r}^{T}}{\partial u_{i}} \frac{\partial}{\partial u_{j}}\left(\frac{\partial \boldsymbol{r}}{\partial t}\right)-\frac{\partial \boldsymbol{r}^{T}}{\partial u_{j}} \frac{\partial}{\partial u_{i}}\left(\frac{\partial \boldsymbol{r}}{\partial t}\right)\right) d \mathcal{V} \\
& +\frac{\partial^{2} D}{\partial \dot{u}_{i} \partial \dot{u}_{j}} \\
k_{i j} & =\int_{\mathcal{V}} \rho \frac{\partial}{\partial u_{j}}\left(\frac{\partial \boldsymbol{r}^{T}}{\partial u_{i}} \frac{\partial^{2} \boldsymbol{r}}{\partial t^{2}}\right) d \mathcal{V}+\frac{\partial^{2} V}{\partial u_{i} \partial u_{j}},
\end{aligned}
$$

where all derivatives are evaluated at $\boldsymbol{u}=\boldsymbol{u}_{0}$ and $D$ is Rayleigh's dissipation function. Note that only the partial time derivatives of the position vector are needed, not the full velocity or acceleration vectors.

Let $\boldsymbol{u}_{\mathrm{g}}$ denote the DOFs for the ground-fixed substructure and $\boldsymbol{u}_{\mathrm{b}_{k}}$ denote the DOFs for rotating blade number $k$ (blades in this paper are always numbered in the direction of the rotation); then the position vector $\boldsymbol{r}$ in the ground-fixed inertial frame to a particle point on the substructure is written as a function of $\boldsymbol{u}_{\mathrm{g}}$ as

$\boldsymbol{r}=\boldsymbol{r}_{\mathrm{g}}\left(\boldsymbol{u}_{\mathrm{g}}\right)$

and on the blade number $k$ as

$\boldsymbol{r}=\boldsymbol{r}_{\mathrm{c}}\left(\boldsymbol{u}_{\mathrm{g}}\right)+\mathbf{T}_{\mathrm{c}}\left(\boldsymbol{u}_{\mathrm{g}}\right)\left(\mathbf{R}_{0}+\mathbf{R}_{1} e^{\iota \psi_{k}}+\overline{\mathbf{R}}_{1} e^{-\iota \psi_{k}}\right) \boldsymbol{r}_{\mathrm{b}}\left(\boldsymbol{u}_{\mathrm{b}_{k}}\right)$,

where the vector $\boldsymbol{r}_{\mathrm{c}}$ and the rotation matrix $\mathbf{T}_{\mathrm{c}}$ describe the position of the rotor center and the orientation of the rotational axis, respectively, both of which are functions of the ground-fixed DOFs $\boldsymbol{u}_{\mathrm{g}}$. The local position vector $\boldsymbol{r}_{\mathrm{b}}$ of a particle on blade number $k$ is a function of $\boldsymbol{u}_{\mathrm{b}_{k}}$, which is the same function for all blades due to the isotropy of the rotor and its discretization. The prescribed rotation of the blade is given by the angle $\psi_{k}=\Omega t+2 \pi(k-1) / B$, where $B$ is the number of blades. The rotation matrix is written in exponential form using a real matrix $\mathbf{R}_{0}$ and a complex matrix $\mathbf{R}_{1}$, which are constant and given by the initial orientation of the rotational axis.

Let the conservative and dissipative forces be linear and depend only on the local DOFs and their time derivatives, 
such that the potential energy and Rayleigh's dissipation function can be written as

$$
\begin{aligned}
& V=\boldsymbol{u}_{\mathrm{g}}^{T} \mathbf{K}_{\mathrm{g}} \boldsymbol{u}_{\mathrm{g}}+\sum_{k=1}^{B} \boldsymbol{u}_{\mathrm{b}_{k}}^{T} \mathbf{K}_{\mathrm{b}} \boldsymbol{u}_{\mathrm{b}_{k}} \\
& \text { and } D=\dot{\boldsymbol{u}}_{\mathrm{g}}^{T} \mathbf{C}_{\mathrm{g}} \dot{\boldsymbol{u}}_{\mathrm{g}}+\sum_{k=1}^{B} \dot{\boldsymbol{u}}_{\mathrm{b}_{k}}^{T} \mathbf{C}_{\mathrm{b}} \dot{\boldsymbol{u}}_{\mathrm{b}_{k}},
\end{aligned}
$$

where $\mathbf{C}_{\mathrm{g}}$ and $\mathbf{K}_{\mathrm{g}}$ are local damping and stiffness matrices for the ground-fixed substructure, and $\mathbf{C}_{\mathrm{b}}$ and $\mathbf{K}_{\mathrm{b}}$ are local and identical damping and stiffness matrices for each blade of the isotropic rotor.

Through insertion of Eq. (10) and the position vectors Eqs. (8) and (9) into the coefficients Eq. (7) and summation over all volumes of the entire structure, the linear equations of motion can be written in a generic matrix form as

$$
\begin{aligned}
{\left[\begin{array}{cc}
\mathbf{M}_{\mathrm{r}} & \mathbf{M}_{\mathrm{gr}}^{T} \\
\mathbf{M}_{\mathrm{gr}} & \mathbf{M}_{\mathrm{g}}
\end{array}\right] \ddot{\boldsymbol{u}}+} & {\left[\begin{array}{cc}
\mathbf{C}_{\mathrm{r}} & \mathbf{G}_{\mathrm{rg}} \\
\mathbf{G}_{\mathrm{gr}} & \mathbf{G}_{\mathrm{g}}+\mathbf{C}_{\mathrm{g}}
\end{array}\right] \dot{\boldsymbol{u}} } \\
+ & {\left[\begin{array}{cc}
\mathbf{K}_{\mathrm{r}}+\mathbf{S}_{\mathrm{r}} & \mathbf{0} \\
\mathbf{S}_{\mathrm{gr}} & \mathbf{K}_{\mathrm{g}}
\end{array}\right] \boldsymbol{u}=\mathbf{0}, }
\end{aligned}
$$

where the $N_{\mathrm{D}}$ DOFs are ordered as

$\boldsymbol{u}=\left\{\boldsymbol{u}_{\mathrm{r}}, \boldsymbol{u}_{\mathrm{g}}\right\}^{T}$ with $\boldsymbol{u}_{\mathrm{r}}=\left\{\boldsymbol{u}_{\mathrm{b}_{1}}, \ldots, \boldsymbol{u}_{\mathrm{b}_{\mathrm{b}}}\right\}^{T}$.

Note that $N_{\mathrm{D}}=N_{\mathrm{g}}+B N_{\mathrm{b}}$, where $N_{\mathrm{g}}$ and $N_{\mathrm{b}}$ are the number of DOFs on the substructure and each blade, respectively. The block matrices of Eq. (11) can be written as

$\mathbf{M}_{\mathrm{r}}=\operatorname{diag}\left\{\mathbf{M}_{\mathrm{b}}, \mathbf{M}_{\mathrm{b}}, \cdots, \mathbf{M}_{\mathrm{b}}\right\}, \mathbf{C}_{\mathrm{r}}=\operatorname{diag}\left\{\mathbf{C}_{\mathrm{b}}, \mathbf{C}_{\mathrm{b}}, \cdots, \mathbf{C}_{\mathrm{b}}\right\}$,

$\mathbf{K}_{\mathrm{r}}=\operatorname{diag}\left\{\mathbf{K}_{\mathrm{b}}, \mathbf{K}_{\mathrm{b}}, \cdots, \mathbf{K}_{\mathrm{b}}\right\}, \mathbf{S}_{\mathrm{r}}=\operatorname{diag}\left\{\mathbf{S}_{\mathrm{b}}, \mathbf{S}_{\mathrm{b}}, \cdots, \mathbf{S}_{\mathrm{b}}\right\}$,

$\mathbf{M}_{\mathrm{g}}(t)=\mathbf{M}_{\mathrm{g}, 0}+\mathbf{M}_{\mathrm{g}, 2} e^{i 2 \Omega t}+\overline{\mathbf{M}}_{\mathrm{g}, 2} e^{-i 2 \Omega t}$

$\mathbf{G}_{\mathbf{g}}(t)=\mathbf{G}_{\mathrm{g}, 0}+\mathbf{G}_{\mathrm{g}, 2} e^{t 2 \Omega t}+\overline{\mathbf{G}}_{\mathrm{g}, 2} e^{-l 2 \Omega t}$

$\mathbf{M}_{\mathrm{gr}}(t)=\mathbf{M}_{\mathrm{gr}, 0}+\mathbf{M}_{\mathrm{gr}, 1} e^{\iota \Omega t}+\overline{\mathbf{M}}_{\mathrm{gr}, 1} e^{-\imath \Omega t}$,

$\mathbf{G}_{\mathrm{gr}}(t)=\mathbf{G}_{\mathrm{gr}, 0}+\mathbf{G}_{\mathrm{gr}, 1} e^{\imath \Omega t}+\overline{\mathbf{G}}_{\mathrm{gr}, 1} e^{-\iota \Omega t}$,

$\mathbf{G}_{\mathrm{rg}}(t)=\mathbf{G}_{\mathrm{rg}, 1} e^{\imath \Omega t}+\overline{\mathbf{G}}_{\mathrm{rg}, 1} e^{-\imath \Omega t}$,

$\mathbf{S}_{\mathrm{gr}}(t)=\mathbf{S}_{\mathrm{gr}, 1} e^{\imath \Omega t}+\overline{\mathbf{S}}_{\mathrm{gr}, 1} e^{-\imath \Omega t}$,

where the time-dependent coupling matrices can be subdivided further into constant single blade components as

$$
\begin{aligned}
\mathbf{M}_{\mathrm{gr}, 0} & =\left[\mathbf{M}_{\mathrm{gb}, 0} \mathbf{M}_{\mathrm{gb}, 0} \cdots \mathbf{M}_{\mathrm{gb}, 0}\right], \\
\mathbf{M}_{\mathrm{gr}, 1} & =\left[\mathbf{M}_{\mathrm{gb}, 1} \mathbf{M}_{\mathrm{gb}, 1} e^{i 2 \pi / B} \cdots \mathbf{M}_{\mathrm{gb}, 1} e^{i 2 \pi(B-1) / B}\right], \\
\mathbf{G}_{\mathrm{gr}, 0} & =\left[\mathbf{G}_{\mathrm{gb}, 0} \mathbf{G}_{\mathrm{gb}, 0} \cdots \mathbf{G}_{\mathrm{gb}, 0}\right], \\
\mathbf{G}_{\mathrm{gr}, 1} & =\left[\mathbf{G}_{\mathrm{gb}, 1} \mathbf{G}_{\mathrm{gb}, 1} e^{i 2 \pi / B} \cdots \mathbf{G}_{\mathrm{gb}, 1} e^{i 2 \pi(B-1) / B}\right], \\
\mathbf{G}_{\mathrm{rg}, 1} & =\left[\mathbf{G}_{\mathrm{bg}, 1}^{T} \mathbf{G}_{\mathrm{bg}, 1}^{T} e^{i 2 \pi / B} \cdots \mathbf{G}_{\mathrm{bg}, 1}^{T} e^{i 2 \pi(B-1) / B}\right]^{T}, \\
\mathbf{S}_{\mathrm{gr}, 1} & =\left[\mathbf{S}_{\mathrm{gb}, 1} \mathbf{S}_{\mathrm{gb}, 1} e^{i 2 \pi / B} \cdots \mathbf{S}_{\mathrm{gb}, 1} e^{i 2 \pi(B-1) / B}\right] .
\end{aligned}
$$

The matrices in Eqs. (13) and (14) related to inertia forces are listed in Appendix A. Note that the 2/rev components of the mass and gyroscopic matrices for the ground-fixed substructure only exist in the case of a two-bladed rotor.

\subsection{Inversion of mass matrix}

The inverse of the mass matrix in Eq. (11) can be written as

$\mathbf{M}^{-1}=\left[\begin{array}{cc}\mathbf{E} & \mathbf{F}^{T} \\ \mathbf{F} & \mathbf{H}\end{array}\right]$,

where

$$
\begin{aligned}
& \mathbf{E}=\mathbf{M}_{\mathrm{r}}^{-1}+\mathbf{M}_{\mathrm{r}}^{-1} \mathbf{M}_{\mathrm{gr}}^{T} \mathbf{H} \mathbf{M}_{\mathrm{gr}} \mathbf{M}_{\mathrm{r}}^{-1}, \mathbf{F}=-\mathbf{H} \mathbf{M}_{\mathrm{gr}} \mathbf{M}_{\mathrm{r}}^{-1}, \\
& \mathbf{H}=\left(\mathbf{M}_{\mathrm{g}}-\mathbf{M}_{\mathrm{gr}} \mathbf{M}_{\mathrm{r}}^{-1} \mathbf{M}_{\mathrm{gr}}^{T}\right)^{-1} .
\end{aligned}
$$

Using Eqs. (13) and (14), it can be shown that the inverse of $\mathbf{H}$ can be written as

$$
\mathbf{H}^{-1}=\mathbf{Q}_{0}+\mathbf{Q}_{2} e^{\imath 2 \Omega t}+\overline{\mathbf{Q}}_{2} e^{-\imath 2 \Omega t},
$$

where the mean and 2/rev components for a $B$-bladed isotropic rotor are

$$
\begin{aligned}
\mathbf{Q}_{0} & =\mathbf{M}_{\mathrm{g}, 0}-B\left(\mathbf{M}_{\mathrm{gb}, 0} \mathbf{M}_{\mathrm{b}}^{-1} \mathbf{M}_{\mathrm{gb}, 0}^{T}+\overline{\mathbf{M}}_{\mathrm{gb}, 1} \mathbf{M}_{\mathrm{b}}^{-1} \mathbf{M}_{\mathrm{gb}, 1}^{T}\right. \\
& \left.+\mathbf{M}_{\mathrm{gb}, 1} \mathbf{M}_{\mathrm{b}}^{-1} \overline{\mathbf{M}}_{\mathrm{gb}, 1}^{T}\right), \\
\mathbf{Q}_{2} & =\left\{\begin{array}{ll}
\mathbf{M}_{\mathrm{g}, 2}-2 \mathbf{M}_{\mathrm{gb}, 1} \mathbf{M}_{\mathrm{b}}^{-1} \mathbf{M}_{\mathrm{gb}, 1}^{T} & \text { for } B=2 \\
0 & \text { for } B>2
\end{array} .\right.
\end{aligned}
$$

Thus, $\mathbf{H}=\mathbf{Q}_{0}^{-1}$ is a real, symmetric, and constant matrix for isotropic rotors with more than two blades. Using Eqs. (16) and (13), this property implies that the inverse mass matrix of such rotors have a finite Fourier series with $2 / \mathrm{rev}$ as the highest harmonic component. Note that the second harmonic component for two-bladed rotors is also symmetric, $\mathbf{Q}_{2}^{T}=\mathbf{Q}_{2}$.

For two-bladed rotors, $\mathbf{H}^{-1}$ is periodic and $\mathbf{H}$ can therefore be written as a Fourier series:

$$
\mathbf{H}=\sum_{n=-\infty}^{\infty} \mathbf{H}_{n} e^{i n \Omega t}
$$

where $\mathbf{H}_{-n}=\overline{\mathbf{H}}_{n}$. Insertion into the equation $\mathbf{H}^{-1} \mathbf{H}=\mathbf{I}$ and collection of terms of equal harmonics yields

$\mathbf{Q}_{0} \mathbf{H}_{0}+\overline{\mathbf{Q}}_{2} \mathbf{H}_{2}+\mathbf{Q}_{2} \overline{\mathbf{H}}_{2}=\mathbf{I}$ mean terms,

$\mathbf{Q}_{0} \mathbf{H}_{1}+\overline{\mathbf{Q}}_{2} \mathbf{H}_{3}+\mathbf{Q}_{2} \overline{\mathbf{H}}_{1}=\mathbf{0} 1 /$ rev terms,

$\mathbf{Q}_{0} \mathbf{H}_{2 m+1}+\overline{\mathbf{Q}}_{2} \mathbf{H}_{2 m+3}+\mathbf{Q}_{2} \mathbf{H}_{2 m-1}=\mathbf{0}(2 m+1) /$ rev terms,

$\mathbf{Q}_{0} \mathbf{H}_{2 m}+\overline{\mathbf{Q}}_{2} \mathbf{H}_{2 m+2}+\mathbf{Q}_{2} \mathbf{H}_{2 m-2}=\mathbf{0}(2 m) /$ rev terms,

where $m=1,2, \ldots$ is a positive integer. The equations for odd terms are homogeneous and regular; thus, all odd harmonic components vanish $\mathbf{H}_{2 m-1}=\mathbf{0}$ for $m=1,2, \ldots$ 
To solve the equations for the even terms, the mean component $\mathbf{H}_{0}$ is obtained from Eq. (21a) as a linear function of the second harmonic component $\mathbf{H}_{2}$ and a constant matrix:

$\mathbf{H}_{0}=\mathbf{Q}_{0}^{-1}-\mathbf{Q}_{0}^{-1}\left(\overline{\mathbf{Q}}_{2} \mathbf{H}_{2}+\mathbf{Q}_{2} \overline{\mathbf{H}}_{2}\right)$.

The remaining even equations can be solved recursively for $\mathbf{H}_{2 m}$ by insertion of the solution for $\mathbf{H}_{2 m-2}$ into the $2 \mathrm{~m} / \mathrm{rev}$ equation. It is convenient to split the equations into real and imaginary parts and solve for each part to obtain

$$
\begin{aligned}
{\left[\begin{array}{c}
\operatorname{Re}\left(\mathbf{H}_{2 m}\right) \\
\operatorname{Im}\left(\mathbf{H}_{2 m}\right)
\end{array}\right] } & =\left(\prod_{k=1}^{m}\left(-\mathbf{P}_{k} \mathbf{Q}_{\mathrm{s}}^{T}\right)\right) \mathbf{B}_{0} \\
& -\mathbf{P}_{m} \mathbf{Q}_{\mathrm{s}}\left[\begin{array}{c}
\operatorname{Re}\left(\mathbf{H}_{2 m+2}\right) \\
\operatorname{Im}\left(\mathbf{H}_{2 m+2}\right)
\end{array}\right] \text { for } m=1,2, \ldots,
\end{aligned}
$$

where the following real matrices have been introduced,

$\mathbf{Q}_{\mathrm{s}}=\left[\begin{array}{cc}\operatorname{Re}\left(\mathbf{Q}_{2}\right) & \operatorname{Im}\left(\mathbf{Q}_{2}\right) \\ -\operatorname{Im}\left(\mathbf{Q}_{2}\right) & \operatorname{Re}\left(\mathbf{Q}_{2}\right)\end{array}\right], \quad \mathbf{B}_{0}=\left[\begin{array}{c}\mathbf{Q}_{0}^{-1} \\ \mathbf{0}\end{array}\right]$,

and the recursive matrices

$\mathbf{P}_{k}=\left(\mathbf{I}-\mathbf{Q}_{\mathrm{d}}^{-1} \mathbf{Q}_{\mathrm{s}}^{T} \mathbf{P}_{k-1} \mathbf{Q}_{\mathrm{s}}\right)^{-1} \mathbf{Q}_{\mathrm{d}}^{-1}$ for $k=1,2, \ldots$,

where $\mathbf{Q}_{\mathrm{d}}=\operatorname{diag}\left\{\mathbf{Q}_{0}, \mathbf{Q}_{0}\right\}$ and $\mathbf{P}_{0}=\operatorname{diag}\left\{2 \mathbf{Q}_{0}^{-1}, \mathbf{0}\right\}$, are $2 \times 2$ block diagonal matrices. Symmetries of $\mathbf{Q}_{0}$ and $\mathbf{Q}_{2}$ and the resulting antisymmetry of $\mathbf{Q}_{\mathrm{s}}$ causes the matrices $\mathbf{P}_{k}$ to be symmetric. Note that $\mathbf{Q}_{d}$ is a regular matrix due to the positive definiteness of the mass matrix.

If $\left\|\mathbf{Q}_{\mathrm{d}}^{-1} \mathbf{Q}_{\mathrm{s}}^{T} \mathbf{P}_{k-1} \mathbf{Q}_{\mathrm{s}}\right\|<1$ then an inequality for the $p$ norm of the product $\mathbf{P}_{k} \mathbf{Q}_{\mathrm{s}}^{T}$ can be derived (Golub and Van Loan, 1996) as

$$
\left\|\mathbf{P}_{k} \mathbf{Q}_{\mathrm{s}}^{T}\right\| \leq \frac{\left\|\mathbf{Q}_{\mathrm{d}}^{-1} \mathbf{Q}_{\mathrm{s}}^{T}\right\|}{1-\left\|\mathbf{Q}_{\mathrm{d}}^{-1} \mathbf{Q}_{\mathrm{s}}^{T} \mathbf{P}_{k-1} \mathbf{Q}_{\mathrm{s}}\right\|} \text { for } k=1,2, \ldots,
$$

where $\|\cdot\|$ here and subsequently denotes any $p$-norm of a matrix. The condition $\left\|\mathbf{Q}_{\mathrm{d}}^{-1} \mathbf{Q}_{\mathrm{s}}^{T} \mathbf{P}_{k-1} \mathbf{Q}_{\mathrm{s}}\right\|<1$ is fulfilled for $k \geq 1$ if $\left\|\mathbf{Q}_{\mathrm{d}}^{-1} \mathbf{Q}_{\mathrm{s}}^{T} \mathbf{P}_{0} \mathbf{Q}_{\mathrm{s}}\right\|<1$. It is not straightforward to prove this inequality, but its validity for a given model can easily be checked. Intuitively, the $p$-norm of the constant part $\mathbf{Q}_{0}$ of the mass matrix for the ground-fixed coordinates should be much larger then the $p$-norm of the second-order harmonic part $\mathbf{Q}_{2}$. It is therefore also assumed that $\left\|\mathbf{P}_{k} \mathbf{Q}_{\mathrm{s}}^{T}\right\|<1$ for $k=1,2, \ldots$ based on the inequality Eq. (26). From the recursive solution Eq. (23), this assumption is sufficient (but not necessary) to ensure that the $p$-norm of harmonic components $\mathbf{H}_{2 m}$ decreases with their order:

$$
\begin{aligned}
\left\|\mathbf{H}_{2 m}\right\| & \leq\left(\prod_{k=1}^{m}\left\|\mathbf{P}_{k} \mathbf{Q}_{\mathrm{s}}^{T}\right\|\right)\left\|\mathbf{B}_{0}\right\|+\left\|\mathbf{P}_{m} \mathbf{Q}_{\mathrm{s}}\right\|\left\|\mathbf{H}_{2 m+2}\right\| \\
& \rightarrow 0 \text { for } m \rightarrow \infty .
\end{aligned}
$$

Thus, closure to the recursive equation (Eq. 23) can therefore be obtained by choosing $\mathbf{H}_{2 N_{\mathrm{h}}+2}=\mathbf{0}$, where $2 N_{\mathrm{h}}$ is the highest harmonic component used in the Fourier series (Eq. 20). The recursive solution of Eq. (23) is computed backward starting with $\mathbf{H}_{2 N_{\mathrm{h}}}$ and ending with $\mathbf{H}_{0}$ given by Eq. (22).

Truncation of Eq. (20) to $2 N_{\mathrm{h}}$ and insertion into Eq. (16) shows that the block matrices of the inverse mass matrix are

$$
\begin{aligned}
& \mathbf{E}=\sum_{n=-\left(2 N_{\mathrm{h}}+2\right)}^{2 N_{\mathrm{h}}+2} \mathbf{E}_{n} e^{i n \Omega t}, \mathbf{F}=\sum_{n=-\left(2 N_{\mathrm{h}}+1\right)}^{2 N_{\mathrm{h}}+1} \mathbf{F}_{n} e^{i n \Omega t}, \text { and } \\
& \mathbf{H}=\sum_{m=-N_{\mathrm{h}}}^{N_{\mathrm{h}}} \mathbf{H}_{2 m} e^{i 2 m \Omega t},
\end{aligned}
$$

where the component matrices $\mathbf{E}_{n}$ and $\mathbf{F}_{n}$ are written out in Appendix B. Thus, the highest order of the harmonics in the inverse mass matrix for a two-bladed rotor is $2 N_{\mathrm{h}}+2$ and involves only the rotor coordinates.

\subsection{Harmonic components in system matrix}

Insertion of Eq. (15) with Eq. (28) into the system matrix Eq. (2) with the gyroscopic/damping and stiffness matrices of Eq. (11) shows that the Fourier series of the periodic system matrix Eq. (3) can be truncated to the order $N$ as

$\mathbf{A}(t)=\sum_{n=-N}^{N} \mathbf{A}_{n} e^{i n \Omega t}$

where

$N= \begin{cases}2 N_{\mathrm{h}}+3 & \text { for } B=2 \\ 3 & \text { for } B>2,\end{cases}$

and the matrices $\mathbf{A}_{n}$ can be found in Appendix C. This analytical derivation of the system matrix is exact for isotropic rotors with more than two blades. In fact, if the anisotropy of the rotor is only related to the stiffnesses of the blades and not the mass distributions or rotor geometry, as in previous studies (e.g., Skjoldan and Hansen, 2009; Skjoldan, 2009), then the system matrices for such rotors also have finite Fourier series. For two-bladed isotropic rotors, the series converges when the Fourier series of the inverse mass matrix converges; sufficient but not necessary criteria for convergence are given in the previous section.

\section{Modal analysis using Hill's method}

This section contains a description of Hill's method and how it can be applied for modal analysis of structures with bladed rotors. First, the concept of periodic mode shapes and Hill's truncated eigenvalue problem is introduced. Then, a novel method for automatic identification of the principal solutions among all eigen-solutions of this eigenvalue problem is briefly presented. The section ends with a description of the modes of bladed rotors, including the identification of the different rotor mode components based on the periodic eigenvectors of Hill's eigenvalue problem. 


\subsection{Periodic mode shapes and Hill's truncated eigenvalue problem}

Floquet theory defines that the eigen-solution of the linear periodic system Eq. (1) consists of an eigenvalue and a corresponding periodic eigenvector. A homogeneous solution to Eq. (1) can therefore be written as

$$
\boldsymbol{x}=\sum_{m=-\infty}^{\infty} \boldsymbol{v}_{m} e^{\imath m \Omega t} e^{\lambda t}
$$

where $\boldsymbol{v}_{m}$ are harmonic components of the periodic eigenvector and $\lambda$ is the complex eigenvalue. Insertion into Eq. (1) with the $2 N_{\mathrm{D}} \times 2 N_{\mathrm{D}}$ periodic system matrix written as the infinite Fourier series (Eq. 3) and collecting terms of equal harmonics yields an infinite set of $2 N_{\mathrm{D}}$ equations:

$$
\sum_{n=-\infty}^{\infty} \mathbf{A}_{n} \boldsymbol{v}_{m-n}-(\lambda+\iota m \Omega) \boldsymbol{v}_{m}=\mathbf{0} \forall m \in \mathbb{Z}
$$

These equations constitute the algebraic eigenvalue problem of infinite dimension that forms the basis of Hill's method (Hill, 1886; $\mathrm{Xu}$ and Gasch, 1995). The eigenvalue is $\lambda$ and the eigenvector can written as $\boldsymbol{v}=\left\{\ldots, \boldsymbol{v}_{-2}^{T}, \boldsymbol{v}_{-1}^{T}, \boldsymbol{v}_{0}^{T}, \boldsymbol{v}_{1}^{T}\right.$, $\left.\boldsymbol{v}_{2}^{T}, \ldots\right\}^{T}$ and is of infinite dimension. If the harmonic index in the eigenvector is shifted by an integer $s$, then the resulting vector $\boldsymbol{v}=\left\{\ldots, \boldsymbol{v}_{-2-s}^{T}, \boldsymbol{v}_{-1-s}^{T}, \boldsymbol{v}_{-s}^{T}, \boldsymbol{v}_{1-s}^{T}, \boldsymbol{v}_{2-s}^{T}, \ldots\right\}^{T}$ and the complex number $\lambda+\iota s \Omega$ are also an eigen-solution. Thus, although the eigenvalue problem has infinitely many eigen-solutions, there are only $2 N_{\mathrm{D}}$ principal solutions from which all other solutions can be constructed. This construction is also clear when inserting the shifted eigen-solution into Eq. (31):

$$
\begin{aligned}
\boldsymbol{x} & =\sum_{m=-\infty}^{\infty} \boldsymbol{v}_{m-s} e^{\imath m \Omega t} e^{(\lambda+\iota s \Omega) t}=\sum_{m=-\infty}^{\infty} \boldsymbol{v}_{m} e^{\iota(m-s) \Omega t} e^{(\lambda+\iota s \Omega) t} \\
& =\sum_{m=-\infty}^{\infty} \boldsymbol{v}_{m} e^{\imath m \Omega t} e^{\lambda t}
\end{aligned}
$$

This arbitrary $s /$ rev shift between the periodicity of the eigenvector and the frequency of the eigenvalue is identical to the frequency indeterminacy in Floquet analysis due to the logarithm of the complex Floquet multipliers (Skjoldan and Hansen, 2009; Bottasso and Cacciola, 2015). The advantage of Floquet analysis is that there are only $2 N_{\mathrm{D}}$ solutions and the frequency indeterminacy can be chosen arbitrarily for each of them; here the concept of participation factors introduced by Bottasso and Cacciola (2015) is helpful. In Hill's method, the problem is to link all eigen-solutions into $2 N_{\mathrm{D}}$ subsets in which a principal eigen-solution can be used to construct all eigen-solutions in the particular subset. A novel method for this linking into subsets and identification of the principal solutions to Hill's eigenvalue problem is presented in the next section.

To numerically solve Hill's eigenvalue problem Eq. (32) is truncated. When the periodic system matrix has a finite
Fourier series (Eq. 29), the periodic eigenvectors can also be represented by a finite Fourier series (Curtis, 2010) in the homogeneous solution

$\boldsymbol{x}=\sum_{m=-M}^{M} \boldsymbol{v}_{m} e^{i m \Omega t} e^{\lambda t}$,

where $M$ is the number of harmonics. The infinite eigenvalue problem Eq. (32) can then be truncated to finite dimension as

$\sum_{n=-N_{m}}^{N_{m}} \mathbf{A}_{n} \boldsymbol{v}_{m-n}-(\lambda+\iota m \Omega) \boldsymbol{v}_{m}=\mathbf{0} \forall m \in[-M: M]$,

where $N_{m}=\min (N,|M-m|)$ is the limit for the summation over the product of the harmonic components of the system matrix and the eigenvector. This limit is lower than the number of harmonics in the system matrix $\mathbf{N}$ for the matrix equations where $|m|>M-N$, showing that truncation errors are introduced in those $2 N$ of the $2 M+1$ matrix equations in Eq. (35). The truncation error has been investigated by Skjoldan (2009) and Lee et al. (2007), who show a convergence of the principal eigen-solutions of the truncated Hill's matrix for their particular systems when $M \geq 2 N$. This finite matrix can be easily set up from Eq. (35) by arranging the harmonic components of the periodic eigenvector as

$\boldsymbol{v}=\left\{\boldsymbol{v}_{-M}^{T}, \ldots, \boldsymbol{v}_{-1}^{T}, \boldsymbol{v}_{0}^{T}, \boldsymbol{v}_{1}^{T}, \ldots, \boldsymbol{v}_{M}^{T}\right\}^{T}$.

The $2 N_{\mathrm{D}}(2 M+1)$ solutions to the truncated eigenvalue problem Eq. (35) will still follow the above rules for index shift, except that the eigen-solutions with the largest harmonic components of their eigenvectors closest to the "edges" at $\pm M$ will be affected by the truncation error. There still exist $2 N_{\mathrm{D}}$ subsets of $2 M+1$ eigen-solutions that can be constructed from a principal solution; however, these solutions with periodic eigenvectors of highest harmonic order will be less accurately constructed. Note that the eigenvector Eq. (36) can be given a norm of 1 , such that $\left\|\boldsymbol{v}_{m}\right\|$ is the participation factor of the $m$ th harmonic component (Bottasso and Cacciola, 2015).

\subsection{Automatic identification of principal solutions}

The identification of the principal solutions can be done manually for small systems (Skjoldan, 2009; Christensen and Santos, 2005; Lee et al., 2007; Kim and Lee, 2012). A more systematic approach is to select the $2 N_{\mathrm{D}}$ eigen-solutions among the $2 N_{\mathrm{D}}(2 M+1)$ solutions that have periodic eigenvectors where the harmonic components are most centered around the mean component; different methods of this concept can be found in Xu and Gasch (1995), Ertz et al. (1995), and Lazarus and Thomas (2010). However, these methods do not always ensure that the identified principal solutions $2 N_{\text {D }}$ can construct all $2 N_{\mathrm{D}}(2 M+1)$ solutions, because two selected principal solutions may come from the same subset, 
whereby one subset is not represented. A novel method is therefore suggested, where the principal solutions are identified in three steps:

1. remove half the eigen-solutions with eigenvectors dominated by harmonic components of the highest order;

2. link the remaining eigen-solutions in $2 N_{\mathrm{D}}$ subsets based on a modal assurance criterion (Allemang, 2003);

3. pick the principal solution in each subset that has the largest mean component $\boldsymbol{v}_{0}$ in its eigenvector.

Step 1 ensures that the eigen-solutions with the largest truncation errors are removed. Step 2 ensures that all $2 N_{\mathrm{D}}$ subsets are represented. The choice of the particular solution in step 3 as the principal one in each subset is less important (similar to Floquet analysis). If the eigenvector with the largest norm of its mean ground-fixed components $\left\|\boldsymbol{v}_{0, \mathrm{~g}}\right\|$ is chosen, then the frequency of the principal eigenvalue will also be the dominant frequency observed in the ground-fixed frame (Skjoldan and Hansen, 2009). For drawing of Campbell diagrams, it is convenient to link the subsets across the variation of rotor speed. Step 3 is therefore only done for one rotor speed computation, and the selection of the principal solutions in each subset for subsequent rotor speeds is based on a modal assurance criterion with the previous speed.

\subsection{Modes of $B$-bladed isotropic rotors}

Modes of a structure with a bladed rotor may be dominated by the motion of the substructure and therefore named after its dominant component of the periodic eigenvector, e.g., "tower fore-aft" or "drivetrain torsion" modes of wind turbines. The name of a rotor mode dominated by blade motion will depend on the number of blades.

The naming conventions of symmetric and whirling rotor modes of three-bladed rotors deduced from the modal analysis using the multi-blade coordinates (Hansen, 2003) can be generalized. For isotropic rotors with more than two blades, the Coleman transformation will render the system matrix time-invariant with constant eigenvectors described in multiblade coordinates (Skjoldan and Hansen, 2009). The Coleman transformation can be written in a complex form, where the cosine and sine parts of the multi-blade coordinates are combined into backward and forward whirling coordinates, similar to complex wave coordinates for vibrations of spinning disks (Lee and Kim, 1995; Hansen, 1999). If we let the modal response in these coordinates be written as $z_{\mathrm{r}} e^{\imath \omega t}$, then the Coleman-transformed responses in rotating blade coordinates will be given as

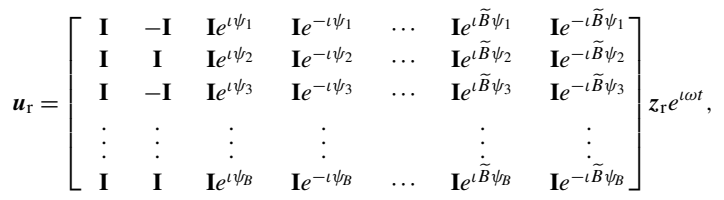

where $\widetilde{B}=(B-1) / 2$ for odd and $\widetilde{B}=B / 2-1$ for an even number of blades, $\psi_{k}=\Omega t+2 \pi(k-1) / B$ is the azimuth angle of blade number $k$, the second column in the matrix is only present for an even number of blades, and the harmonic azimuth dependent parts are omitted for two-bladed rotors. These harmonic parts come in pairs for each harmonic order with plus and minus on the blade azimuth angle, defining the direction of the whirling. The constant eigenvector in complex whirling coordinates can be written as $z_{\mathrm{r}}=\left\{\mathbf{A}_{0}^{T}, \mathbf{A}_{\mathrm{d}}^{T}\right.$, $\left.\mathbf{A}_{\mathrm{BW}, 1}^{T}, \mathbf{A}_{\mathrm{FW}, 1}^{T}, \ldots, \mathbf{A}_{\mathrm{BW}, \widetilde{B}}^{T}, \mathbf{A}_{\mathrm{FW}, \widetilde{B}}^{T}\right\}^{T}$, which by substitution into Eq. (37) shows that the modal response of blade number $k$ of the isotropic rotor can be written as

$$
\begin{aligned}
\boldsymbol{u}_{\mathrm{b}_{k}} & =\left(\mathbf{A}_{0}+(-\mathbf{I})^{k} \mathbf{A}_{\mathrm{d}}\right) e^{\imath \omega t} \\
& +\sum_{p=1}^{\widetilde{B}} \mathbf{A}_{\mathrm{BW}, p} e^{\ell(\omega+p \Omega) t} e^{\ell \frac{2 \pi p(k-1)}{B}} \\
& +\sum_{p=1}^{\widetilde{B}} \mathbf{A}_{\mathrm{FW}, p} e^{\ell(\omega-p \Omega) t} e^{-\imath \frac{2 \pi p(k-1)}{B}},
\end{aligned}
$$

showing that $\mathbf{A}_{0}$ are symmetric components, $\mathbf{A}_{\mathrm{d}}$ are antisymmetric components, and $\mathbf{A}_{\mathrm{BW}, p}$ and $\mathbf{A}_{\mathrm{FW}, p}$ are backward (BW) and forward whirling (FW) components of the blade motion in the mode. The direction of the whirl is given by the sign of the phase shifts $\frac{2 \pi p}{B}(k-1)$ for each blade. Note that for BW components, the angular frequency $p / r e v$ is added to the eigenfrequency $\omega$, and it is subtracted for FW modes. As explained in Hansen (2003, 2007), the eigenfrequencies of a three-bladed rotor system described in multiblade coordinates are measured in the ground-fixed frame, in which the frequencies of a pure BW mode $\omega_{\mathrm{BW}}$ and pure FW mode $\omega_{\mathrm{FW}}$ decrease and increase with $1 / \mathrm{rev}$, respectively, such that their frequencies in the rotating blade frame given by Eq. (38) are close to the frequency of the corresponding blade mode $\omega_{\mathrm{b}} \approx \omega_{\mathrm{BW}}+\Omega \approx \omega_{\mathrm{FW}}-\Omega$. Equation (38) shows that generally there will be whirling mode pairs that split with up to $2 \widetilde{B} / \mathrm{rev}$ in the ground-fixed frame, where the general relationship $\omega_{\mathrm{b}} \approx \omega_{\mathrm{BW}}+p \Omega \approx \omega_{\mathrm{FW}}-p \Omega$ to the blade frequencies holds. Note that the phase speed $\frac{2 \pi p}{B}$ of the rotor whirling will be a factor $p$ higher. Note also that the frequencies of antisymmetric components for an even number of blades (except for two) are unchanged by the transformation from ground-fixed to rotating blade frame. These properties shared by the symmetric components are caused by the fact that the reaction forces due to these components of rotor motion is neutral with respect to the rotation; the center of gravity of the entire rotor is only moved axially by symmetric components and remains stationary for antisymmetric components.

Each harmonic component of each DOF of a rotor mode may therefore be named symmetric, antisymmetric (exists only for an even number of blades), backward whirling (exists only for $B \geq 3$ ), or forward whirling (exists only for 
$B \geq 3$ ), followed by the name of the DOF. For rotors with more than four blades, the whirling components must also have the phase speed index $p=1,2, \ldots \widetilde{B}$ added to the name of the particular component. The symmetric, antisymmetric, and whirling components of the rotor motion given by Eq. (38) can be derived by inverting the transformation Eq. (37). By inserting the rotor motion $\boldsymbol{u}_{\mathrm{r}}=\boldsymbol{v}_{m, \mathrm{r}} e^{i m \Omega t} e^{\lambda t}$ of the $m$ th harmonic of the periodic eigenvector into Eq. (37) and setting the time to zero $t=0$, the symmetric, antisymmetric, and whirling components of this harmonic can be derived as

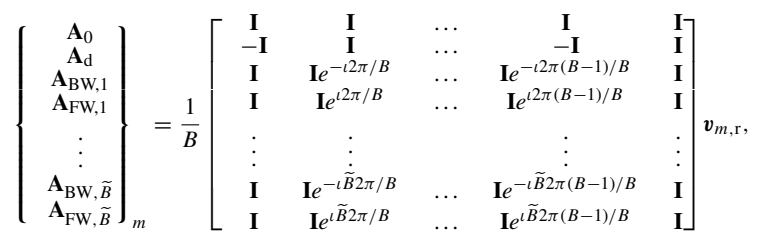

where $\boldsymbol{v}_{m, \mathrm{r}}$ is the rotor part of a harmonic component $\boldsymbol{v}_{m}$ of the eigenvector $\boldsymbol{v}$, and the last row of matrices is omitted for rotors with odd number of blades. The harmonic order $m$ of each component is also relevant for its naming, but we again note that it is directly dependent on the choice of the principal solution. Note that two-bladed rotors only have symmetric and antisymmetric components.

\section{Modal analysis of two- and three-bladed wind turbines}

The theories presented in the previous sections are applicable to structures with isotropic rotors with any number of blades higher than one. In this section, the modal dynamics of twoand three-bladed turbines are investigated because they are of the highest interest to the wind turbine industry, but also because the finite Fourier series of the system matrix shows that there are no qualitative difference between turbines with three or more identical blades.

The turbine used for the analysis is the DTU $10 \mathrm{MW}$ reference wind turbine (RWT) by Bak et al. (2012) with three identical blades. For the sake of comparability of the modal dynamics, the two-bladed version is obtained by reusing the same blade. In reality, the optimal aerodynamic solidity of the rotor would require a redesign (Bergami et al., 2014); the blades would get a larger chord and the increased absolute thickness (assuming the same relative thickness of the airfoils) would be used to either decrease the blade mass, increase the blade stiffness (keeping the same bending stresses) or combine these two objectives. In any case, the blade for the two-bladed turbine will have different blade modal frequencies and possible mass distribution, which would complicate the direct comparison of turbine modes to the threebladed version.

The turbine model derived in the next section is based on the simple model presented in Hansen (2003), except that the bending of the main shaft is omitted here and the generator rotation has been added as a new DOF. The model parameters are tuned such that the modal frequencies of the first 11 modes of the three-bladed turbine are as close as possible to the modal frequencies computed for the $10 \mathrm{MW}$ RWT with the high-fidelity linear model of the software HAWCStab2 using beam elements and the method of the Coleman transformation (Hansen, 2004). Minor modifications of the turbine have been introduced such that the center of gravity in the blade cross sections coincides with the pitch axis and the coning is also set to zero.

The convergence of the Fourier series of the system matrix for the two-bladed turbine is analyzed in Sect. 4.2. The Campbell diagrams of the principal modal frequencies are presented for 3- and two-bladed turbines in Sect. 4.3. The well-known periodic mode shapes of three-bladed turbines are repeated in Sect. 4.4, and more complex periodic mode shapes for two-bladed turbines are presented in Sect. 4.5. The section ends with a discussion of the differences between the modal dynamics of the two turbine types.

\subsection{Model kinematics and parameters}

Figure 1 shows an illustration of the structural turbine model. The nacelle and tower motions are described by five DOFs. The nacelle can translate in the two horizontal directions described in the ground-fixed inertial frame $(X, Y, Z)$ by $u_{x}$ (side-side) and $u_{y}$ (fore-aft). It can tilt, roll, and yaw described by the angles $\theta_{x}, \theta_{y}$, and $\theta_{z}$, respectively. The azimuthal angle of the blade number one is $\psi_{1}=\Omega t+\psi_{\mathrm{s}}+\psi_{\mathrm{g}}$, where $\Omega$ is the constant mean speed and $\psi_{\mathrm{s}}$ and $\psi_{\mathrm{g}}$ are the torsional and rigid-body rotations of the drivetrain, respectively. The generator is rotating at the speed $\Omega+\dot{\psi}_{\mathrm{g}}$.

The blade motion is described in their own rotating frames $(x, y, z)$, where the $z$ axis is the blade axis and the $y$ axis at rest coincides with the $y$ axis. The local position vector for the center of gravity on blade number $k$ is described by an expansion in the first three blade modes at standstill as

$$
\begin{aligned}
& \boldsymbol{r}_{\mathrm{b}}\left(\boldsymbol{u}_{\mathrm{b}_{k}}\right)=\left\{\begin{array}{c}
\phi_{x, \mathrm{f}_{\mathrm{f}}}(z) \\
\phi_{y, \mathrm{f}_{1}}(z) \\
z
\end{array}\right\} q_{\mathrm{f}_{1}, k}(t)+\left\{\begin{array}{c}
\phi_{x, \mathrm{e}}(z) \\
\phi_{y, \mathrm{e}}(z) \\
z
\end{array}\right\} q_{\mathrm{e}, k}(t) \\
& +\left\{\begin{array}{c}
\phi_{x, \mathrm{f}_{2}}(z) \\
\phi_{y, \mathrm{f}_{2}}(z) \\
z
\end{array}\right\} q_{\mathrm{f}_{2}, k}(t)
\end{aligned}
$$

for $z \in\left[R_{\mathrm{h}}: R\right]$ and $\boldsymbol{r}_{\mathrm{b}}=\{0,0, z\}^{T}$ for $z \in\left[0: R_{\mathrm{h}}\right]$, where $R_{\mathrm{h}}$ and $L_{\mathrm{b}}$ are the hub radius and blade length, respectively. The outer rotor radius is $R=R_{\mathrm{h}}+L_{\mathrm{b}}$. The edgewise and flapwise deflections in the first flapwise blade mode are $\phi_{x, f_{1}}(z)$ and $\phi_{y, f_{1}}(z)$, respectively, and $q_{f_{1}, k}$ is the DOF of this deflection shape for blade number $k$. Similar, the subscripts "e" and " $f_{2}$ " denote the contributions from the first edgewise and second flapwise deflection shapes. All shape functions are obtained by polynomial fits to the isolated blade mode shapes computed with the beam element model of HAWCStab2 (see 

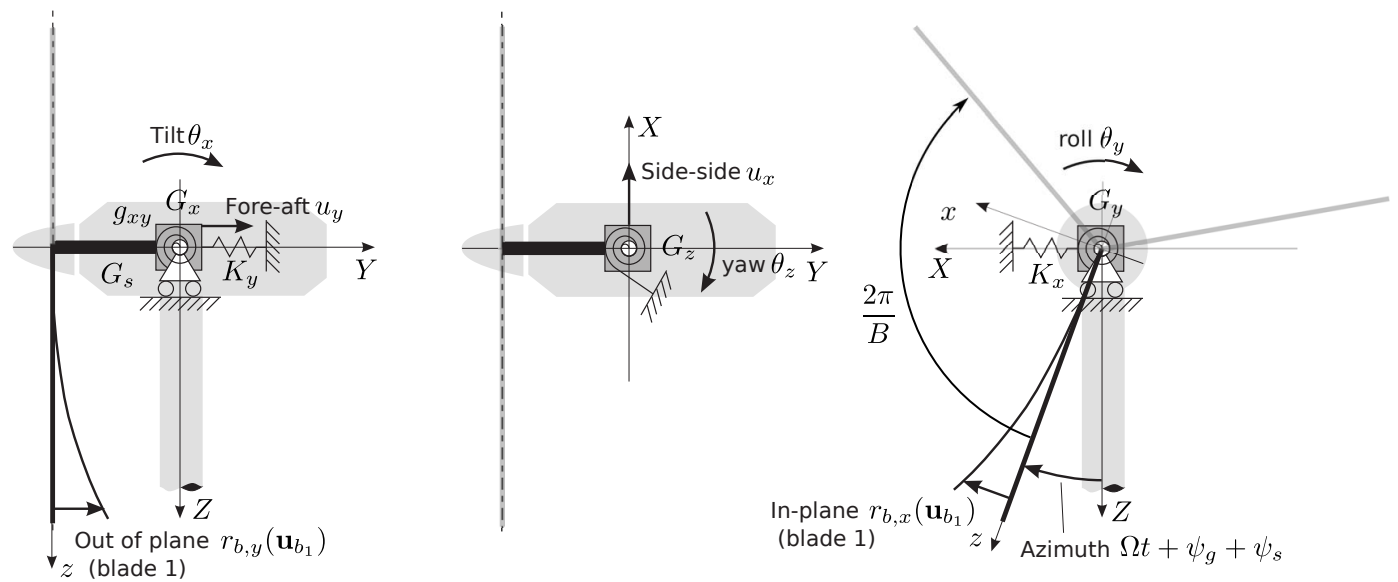

Figure 1. Illustration of the simple turbine model.
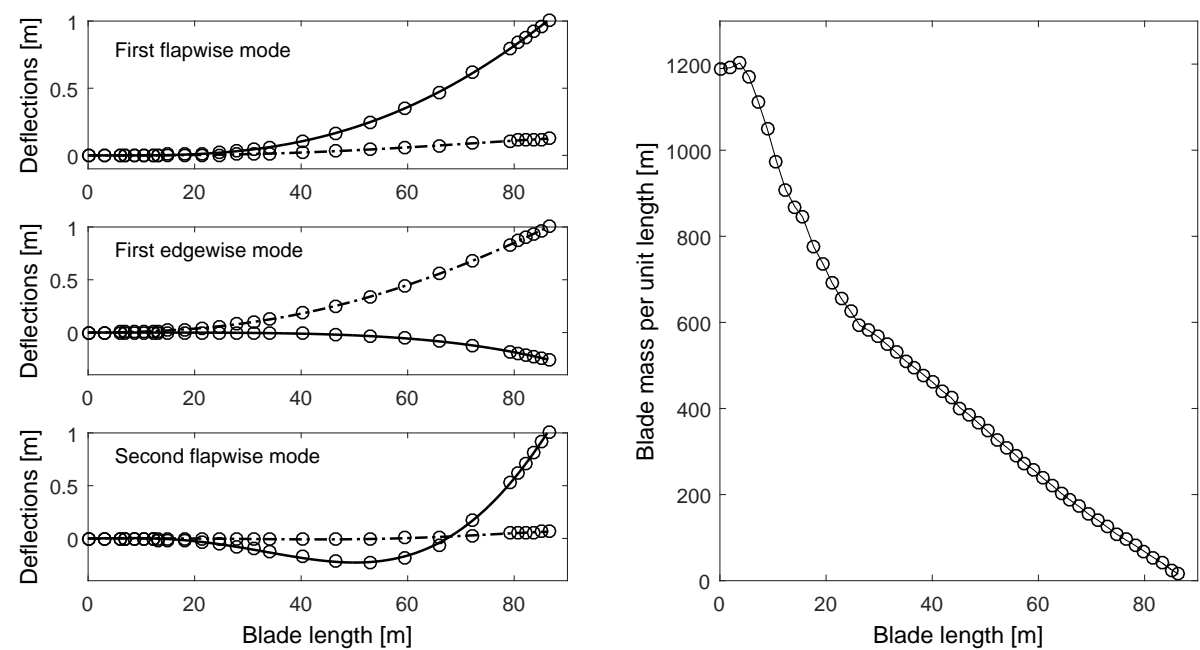

Figure 2. Edgewise (dashed curves) and flapwise (solid curves) deflections in the blade mode shapes (left plots) and the blade mass distribution (right plot) for the blade of the DTU $10 \mathrm{MW}$ RWT. Circles in the left plots are results from the beam element model of the software HAWCStab2 and the curves are polynomial fits used in the present model.

Fig. 2). The vector containing the system DOFs is defined according to Eq. (12) as

$$
\begin{aligned}
\boldsymbol{u}= & \left\{q_{\mathrm{f}_{1}, 1}, q_{\mathrm{e}, 1}, q_{\mathrm{f}_{2}, 1}, \ldots, q_{\mathrm{f}_{1}, B}, q_{\mathrm{e}, B}, q_{\mathrm{f}_{2}, B}, u_{x}, u_{y}, \theta_{x},\right. \\
& \left.\theta_{y}, \theta_{z}, \psi_{\mathrm{g}}, \psi_{\mathrm{s}}\right\}^{T},
\end{aligned}
$$

where the number of DOFs is dependent on the number of blades as $N_{\mathrm{D}}=3 B+7$. To obtain the linear equations of motion using the derivations of Sect. 2.1, the blade mass motion is written in the form of Eq. (9) using Eq. (40) and the following rotor center position and orientation of the rotational axis,

$\boldsymbol{r}_{\mathrm{c}}=\left[\begin{array}{ccc}1 & -\theta_{z} & \theta_{y} \\ \theta_{z} & 1 & -\theta_{x} \\ -\theta_{y} & \theta_{x} & 1\end{array}\right]\left\{\begin{array}{c}0 \\ -L_{\mathrm{s}} \\ 0\end{array}\right\}$

$$
\begin{aligned}
& \mathbf{T}_{\mathrm{c}}=\left[\begin{array}{ccc}
1 & -\theta_{z} & \theta_{y} \\
\theta_{z} & 1 & -\theta_{x} \\
-\theta_{y} & \theta_{x} & 1
\end{array}\right] \\
& {\left[\begin{array}{ccc}
\cos \left(\psi_{\mathrm{s}}+\psi_{\mathrm{g}}\right) & 0 & \sin \left(\psi_{\mathrm{s}}+\psi_{\mathrm{g}}\right) \\
0 & 1 & 0 \\
-\sin \left(\psi_{\mathrm{s}}+\psi_{\mathrm{g}}\right) & 0 & \cos \left(\psi_{\mathrm{s}}+\psi_{\mathrm{g}}\right)
\end{array}\right],}
\end{aligned}
$$

and constant rotation matrices

$$
\mathbf{R}_{0}=\left[\begin{array}{lll}
1 & 0 & 0 \\
0 & 0 & 0 \\
0 & 0 & 1
\end{array}\right] \text { and } \mathbf{R}_{1}=\left[\begin{array}{ccc}
1 / 2 & 0 & -\iota / 2 \\
0 & 0 & 0 \\
\iota / 2 & 0 & 1 / 2
\end{array}\right] \text {. }
$$

These vector and matrix functions of the DOFs are inserted into the volume integrals for the matrix elements in Appendix A, which reduce to line integrals over $z \in[0: R]$. The mass distribution of hub is defined as $m(z)=M_{\mathrm{h}} / B / R_{\mathrm{h}}$ for $z \in\left[0: R_{\mathrm{h}}\right]$, where $M_{\mathrm{h}}$ is the total hub mass. The mass distribution of the blade is plotted in Fig. 2 . 
Table 1. Tuned parameters of simple model to fit the modal properties of the DTU 10 MW RWT up to its 11 th mode.

\begin{tabular}{lll}
\hline Parameter description & Symbol & Value \\
\hline Blade length & $L_{\mathrm{b}}$ & $86.366 \mathrm{~m}$ \\
First blade flap frequency & $\omega_{\mathrm{f}_{1}}$ & $0.610 \mathrm{~Hz}$ \\
First blade edge frequency & $\omega_{\mathrm{e}}$ & $0.934 \mathrm{~Hz}$ \\
Second blade flap frequency & $\omega_{\mathrm{f}_{2}}$ & $1.738 \mathrm{~Hz}$ \\
Hub radius & $R_{\mathrm{h}}$ & $2.8 \mathrm{~m}$ \\
Hub mass & $M_{\mathrm{h}}$ & $105520 \mathrm{~kg}$ \\
Tower to rotor distance & $L_{\mathrm{S}}$ & $7.1 \mathrm{~m}$ \\
Generator inertia on low-speed shaft & $I_{\mathrm{g}}$ & $3751000 \mathrm{~kg} \mathrm{~m}^{2}$ \\
Drivetrain stiffness & $G_{\mathrm{s}}$ & $0.668 \mathrm{GNm} \mathrm{rad}^{-1}$ \\
Nacelle/effective tower mass & $M$ & $446040 \mathrm{~kg}^{2}$ \\
Nacelle tilt inertia & $I_{x}$ & $4106000 \mathrm{~kg} \mathrm{~m}^{2}$ \\
Nacelle roll inertia & $I_{y}$ & $410600 \mathrm{~kg} \mathrm{~m}^{2}$ \\
Nacelle yaw inertia & $I_{z}$ & $4106000 \mathrm{~kg} \mathrm{~m}^{2}$ \\
Tower top side-side stiffness & $K_{x}$ & $7.4 \mathrm{MN} \mathrm{m}^{-1}$ \\
Tower top fore-aft stiffness & $K_{y}$ & $7.4 \mathrm{MN} \mathrm{m}^{-1}$ \\
Tower top tilt stiffness & $G_{x}$ & $7.462 \mathrm{GNm} \mathrm{rad}^{-1}$ \\
Tower top roll stiffness & $G_{y}$ & $7.462 \mathrm{GNm} \mathrm{rad}^{-1}$ \\
Tower top coupling stiffness & $g_{x y}$ & $0.2035 \mathrm{GN}^{-1}$ \\
Tower top yaw stiffness & $G_{z}$ & $3.5 \mathrm{GNm} \mathrm{rad}^{-1}$ \\
\hline
\end{tabular}

The ground-fixed substructure is modeled as a lumped mass, and the inertia forces from the nacelle and effective tower masses and the generator rotational inertia are derived from the kinetic energy:

$T_{\mathrm{g}}=\frac{1}{2} M\left(\dot{u}_{x}+\dot{u}_{y}\right)^{2}+\frac{1}{2} I_{x} \dot{\theta}_{x}^{2}+\frac{1}{2} I_{y} \dot{\theta}_{y}^{2}+\frac{1}{2} I_{z} \dot{\theta}_{z}^{2}+\frac{1}{2} I_{\mathrm{g}} \dot{\psi}_{\mathrm{g}}^{2}$,

such that the first term of $m_{\mathrm{g}, 0, i j}$ in Eq. (A2) is replaced by $\partial^{2} T_{\mathrm{g}} / \partial \dot{u}_{i} \partial \dot{u}_{j}$. The potential energy for the linear elastic stiffnesses of the nacelle/tower motion, shaft torsion, and blade deflections is written as

$$
\begin{gathered}
V=\frac{1}{2} K_{x} u_{x}^{2}+\frac{1}{2} K_{y} u_{y}^{2}+\frac{1}{2} G_{x} \theta_{x}^{2}+\frac{1}{2} G_{y} \theta_{y}^{2}+\frac{1}{2} G_{z} \theta_{z}^{2} \\
-g_{x y} \theta_{x} u_{y}+g_{x y} \theta_{y} u_{x}+\frac{1}{2} G_{\mathrm{s}} \psi_{\mathrm{s}}^{2}+\sum_{k=1}^{B} V_{\mathrm{b}_{k}},
\end{gathered}
$$

where $V_{\mathrm{b}_{k}}$ is the potential energy of blade number $k$ given as

$$
\begin{aligned}
V_{\mathrm{b}_{k}}= & \sum_{\beta=\left[\mathrm{f}_{1}, \mathrm{e}, \mathrm{f}_{2}\right]} \frac{1}{2} M_{\beta} \omega_{\beta}^{2} q_{\beta, k}^{2}+\Omega^{2} \sum_{\beta=\left[\mathrm{f}_{1}, \mathrm{e}, \mathrm{f}_{2}\right]} \int_{0}^{R} \\
& \left(\left(\frac{\partial^{2} \phi_{x, \beta}}{\partial z^{2}}\right)^{2}\left(\frac{\partial^{2} \phi_{y, \beta}}{\partial z^{2}}\right)^{2}\right) q_{\beta, k}^{2} \int_{z}^{R} m(s) \mathrm{d} s \mathrm{~d} z,
\end{aligned}
$$

where $M_{\beta}$ and $\omega_{\beta}$ are the modal mass and frequency of the orthogonal blade modes $\beta=\left[f_{1}, e, f_{2}\right]$. The last term is the potential energy of the centrifugal forces proportional to the squared rotor speed.

The damping matrices of Rayleigh's dissipation function in Eq. (10) for the blades $\mathbf{C}_{\mathrm{b}}$ and the nacelle/tower motion

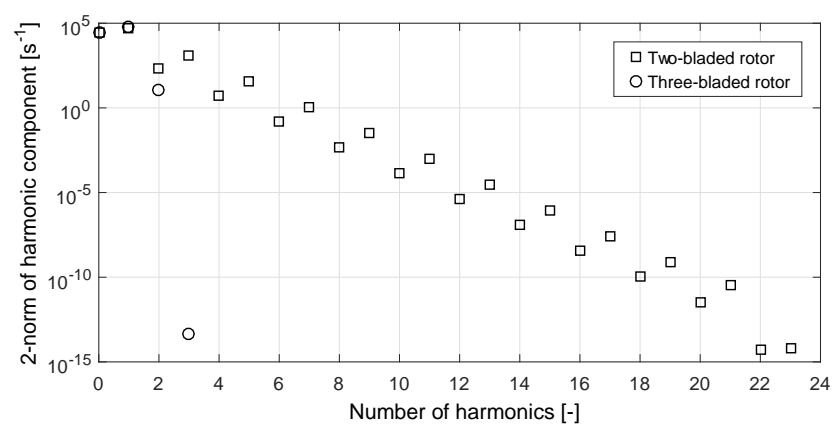

Figure 3. The 2-norms of the Fourier components of the system matrices for the two- and three-bladed simple models of the DTU 10 MW RWT.

and shaft torsion $\mathbf{C}_{\mathrm{g}}$ are set up using a spectral damping model (Clough and Penzien, 1975). The first and second flapwise blade modes are set to have logarithmic decrements of 20 and $10 \%$, respectively, whereas all other modes are damped 2-5\%. These choices of damping are simply crude approximations to the aeroelastic damping of the turbine in operation (Bak et al., 2012), and they have no significant effect on the results of Hill's method. Damping is very important for solutions using Floquet theory where the system is time-integrated.

The linear equations of motion (Eq. 11) can be derived from Eq. (7) and the integrals in Appendix A using the above kinematic description of the blade motion, the kinetic energy of the nacelle/tower and generator inertia, the total potential energy, and the spectral damping matrices. For brevity, the block matrices of Eq. (11) are not explicitly included. All parameters (except for the blade properties in Fig. 2) of the tuned model are listed in Table 1.

\subsection{Convergence of Fourier series for two-bladed rotor}

Convergence of the Fourier series for the system matrix of two-bladed isotropic rotors is ensured if the constant part of the mass matrix for the ground-fixed DOFs is sufficiently larger then the second-order harmonic part. Using Eqs. (24) and (25), it can be computed that $\left\|\mathbf{Q}_{\mathrm{d}}^{-1} \mathbf{Q}_{\mathrm{s}}^{T} \mathbf{P}_{0} \mathbf{Q}_{\mathrm{s}}\right\| \approx 0.22<1$ and $\left\|\mathbf{P}_{k} \mathbf{Q}_{\mathrm{s}}^{T}\right\|<0.67$ for $k=1$, $2, \ldots$, i.e., the inverse mass matrix and therefore the system matrix have finite Fourier series for the two-bladed rotor.

Figure 3 shows the 2-norms of the Fourier components of the system matrices for the models with the two- and threebladed isotropic rotors. The convergence for the two-bladed rotor is observed and for the following analysis the number of harmonics is chosen to be $N=7$. The largest norm for the $1 / \mathrm{rev}$ component is $46480 \mathrm{~s}^{-1}$ and the largest norm of an omitted component $(9 / \mathrm{rev})$ is $0.03 \mathrm{~s}^{-1}$. For the three-bladed rotor all $N=3$ harmonic components are used although the third harmonic is effectively zero. 

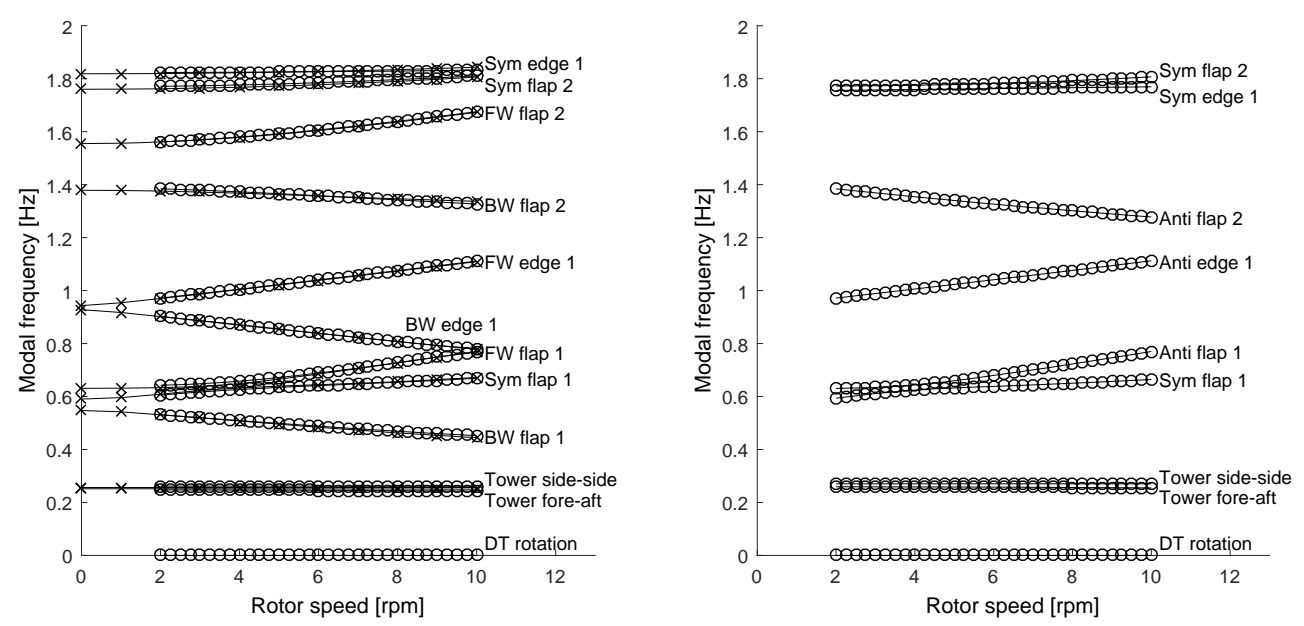

Figure 4. Campbell diagrams of the principal modal frequencies of the three-bladed (left plot) and two-bladed (right plot) version of the DTU 10 MW RWT computed with Hill's method (circles) and with the software HAWCStab2 (crosses) for the three-bladed turbine.

\subsection{Campbell diagrams of two- and three-bladed turbines}

The number of harmonics in the periodic eigenvectors used for Hill's truncated eigenvalue problem is set to $M=2 N$ for both rotors; thus, $M=14$ and $M=6$ for the two- and threebladed rotor, respectively. The eigenvalue problem is solved for 33 equidistant rotor speeds ranging from 2 to $10 \mathrm{rpm}$ (nominal speed of the DTU 10 MW RWT is $9.6 \mathrm{rpm}$ ). The principal solutions at the lowest speed of $2 \mathrm{rpm}$ are selected among the subsets of solutions as the one that has the largest mean component on the ground-fixed DOFs (see Sect. 3.2). For the remaining rotor speeds, the principal solutions are selected as the ones that have the largest modal assurance criterion relative to the principal solutions selected at the previous rotor speed.

Figure 4 shows the Campbell diagrams of principal modal frequencies versus rotor speed for the three-bladed (left) and two-bladed (right) versions of the DTU $10 \mathrm{MW}$ RWT. The mode names are deduced from the dominant components in the periodic mode shapes (see Sects. 4.4 and 4.5). For the three-bladed rotor, the principal modal frequencies computed with Hill's method (circles) are in close agreement with the modal frequencies computed with the high-fidelity software HAWCStab2 (crosses). This agreement shows that the simple model combined with the automatic identification of the principal solutions is able to predict the same modal frequencies in the ground-fixed frame as computed using the Coleman transformation method.

Comparison of the two Campbell diagrams shows that the tower modes of the two-bladed turbine have slightly higher frequencies due to the lighter rotor. The $\pm 1 /$ rev frequency splitting for the pairs of whirling rotor modes in the groundfixed frame is clear for the three-bladed turbine (noting that out-of-plane blade deflections are stiffened by the centrifugal forces). For the two-bladed turbine, there are no whirling modes but the antisymmetric modes are still either increasing (first edge and flap) or decreasing (second flap) with $1 / \mathrm{rev}$, because the principal solutions are selected as the solutions with the largest mean components on ground-fixed DOFs. Campbell diagrams containing only the principal frequencies are not very informative for two-bladed turbines. Instead, the periodic mode shapes of the selected principal solutions are now analyzed. Note that first mode of both turbines is the trivial rigid-body rotation of the drivetrain, which is omitted from this analysis.

\subsection{Periodic mode shapes of three-bladed turbines}

Figures 5-15 show the dominant modal components of selected DOFs in the periodic mode shapes of the three-bladed turbine. The modal amplitudes are plotted as functions of both the rotor speed $\Omega$ and the frequency of the particular component $\omega_{0}+m \Omega$, where $\omega_{0}$ is the principal modal frequency and $m$ is the harmonic order of the component (see Eq. 34). Only amplitudes higher than $10 \%$ of the overall maximum amplitude across all rotor speeds are plotted. The generator rotation and shaft torsion angles are multiplied by 10 for better scaling of these small angles compared to blade deflections. Scaling of the tower translations are also applied to show weak couplings for some modes. The plot layout is the same in all figures: a three-dimensional plot in the top and its projections onto the amplitude-rotor speed (left) and frequency-rotor speed (right) planes in the lower plots. The principal frequencies $\omega_{0}$ are always denoted by black filled circles in the frequency-rotor speed planes. The color coding of the modal components is also the same in all figures: black markers denote amplitudes of ground-fixed DOFs. Red, green, and blue markers denote amplitudes of the rotor components of the first flapwise, first edgewise, and second flapwise deflection shapes, respectively, computed using Eq. (39). Note that the frequency-rotor speed diagrams 

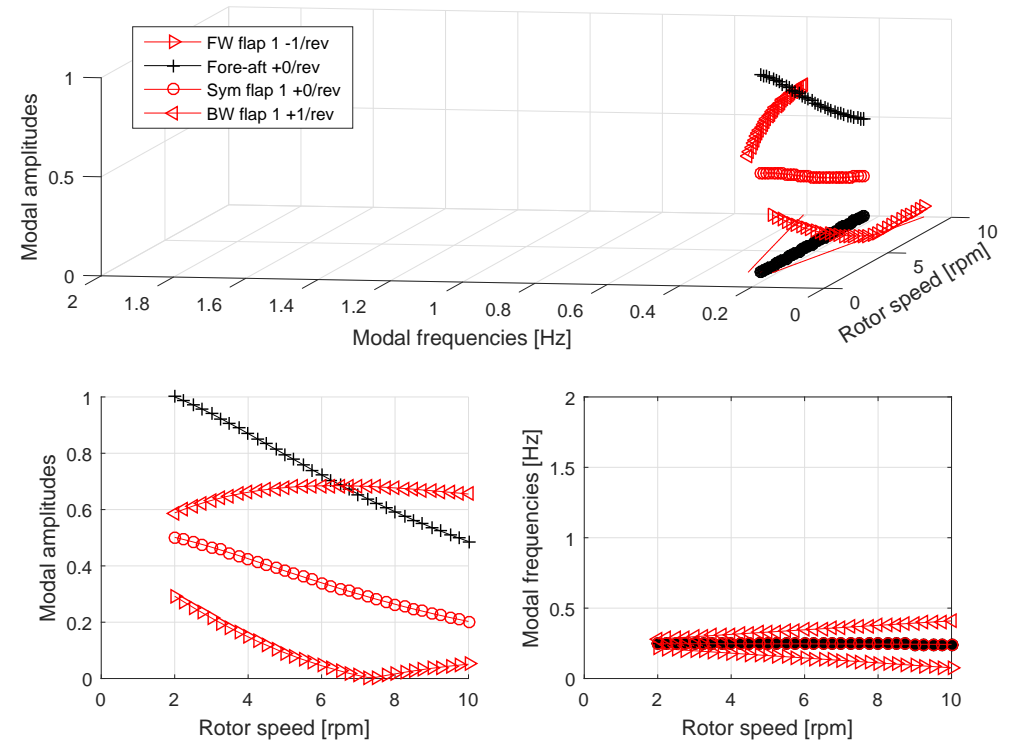

Figure 5. Harmonic modal components for the three-bladed turbine mode dominated by tower fore-aft motion. Top panel: modal amplitudes plotted versus rotor speed and frequency of the particular harmonic component. Lower left panel: projection onto the plane of modal amplitudes and rotor speed. Lower right panel: projection onto the plane of component frequencies and rotor speed (periodic Campbell diagram). Filled black circles show the principal modal frequencies in the frequencies and rotor speed planes. Only modal components with amplitudes larger than $10 \%$ of the overall maximum amplitude are plotted.
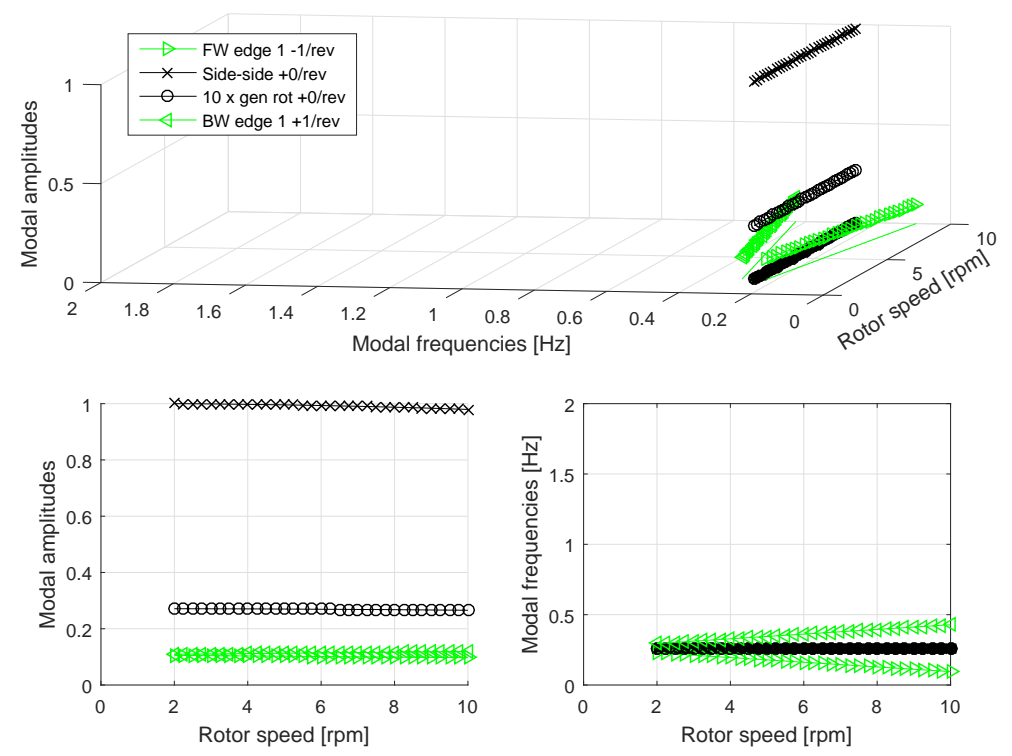

Figure 6. Harmonic modal components for the three-bladed turbine mode dominated by tower side-side motion. Plot layout as in Fig. 5.

(lower right) are similar to the periodic Campbell diagrams in Bottasso and Cacciola (2015), except that each curve represents the magnitude of the harmonic component for a particular DOF and not the vector norm of all DOFs like the participation factors.

The naming of the modes shown in Fig. 4 is deduced from the harmonic components that dominate the periodic mode shapes observed in Figs. 5-15 across a large part of the rotor speed range. Note that the first flapwise symmetric and forward whirling modes interchange their mode shapes at low rotor speeds. The routine for sorting the modes based on a modal assurance criterion cannot always distinguish strongly coupled modes from each other. This issue is dependent on the resolution of the rotor speed range.

A general observation for the three-bladed turbine modes is that no harmonic component of the rotor response is 

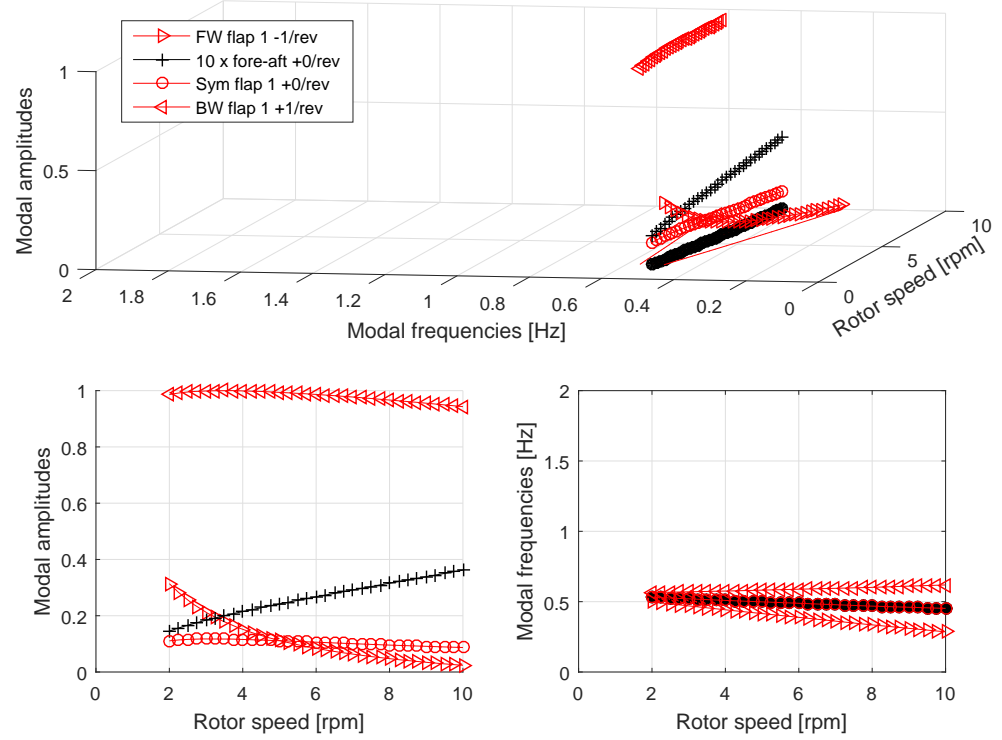

Figure 7. Harmonic modal components for the three-bladed turbine mode dominated by backward whirling (BW) of the rotor in the first flapwise DOF. Plot layout as in Fig. 5.
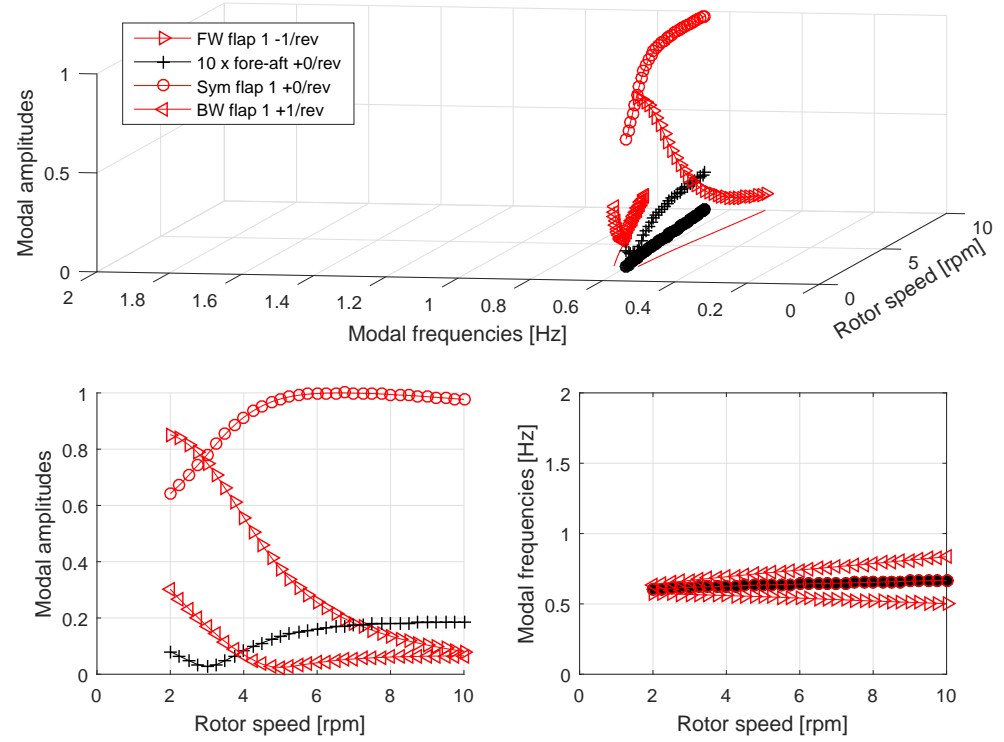

Figure 8. Harmonic modal components for the three-bladed turbine mode dominated by symmetric deflection of the rotor in the first flapwise DOF across the rotor speed range. Plot layout as in Fig. 5.

more than $\pm 1 /$ rev from the principal frequency. Lowering the threshold for the plotted amplitudes to $0.1 \%$ of the overall maximum amplitude did not change this observation. This agrees with Eq. (38) and previous studies of isotropic threebladed turbines using the Coleman transformation method (Hansen, 2003, 2007): an inverse Coleman transformation from the ground-fixed frame with a single modal frequency back to the rotating frame shows that symmetric components will have the same frequency as in the ground-fixed frame, but $\mathrm{BW}$ and FW components will have frequencies that are
$+1 /$ rev and $-1 /$ rev, respectively, from a principal frequency defined in the ground-fixed frame. If the three-bladed rotor was anisotropic, then secondary harmonics will arise whose magnitudes will depend on the magnitude of the anisotropy (Skjoldan and Hansen, 2009).

Looking at the individual mode shapes, there are couplings of the different DOFs in each mode of three-bladed turbines. The tower fore-aft mode (Fig. 5) contains symmetric, BW, and FW components in the first flapwise blade mode. Similarly, the tower side-side mode (Fig. 6) contains BW and 

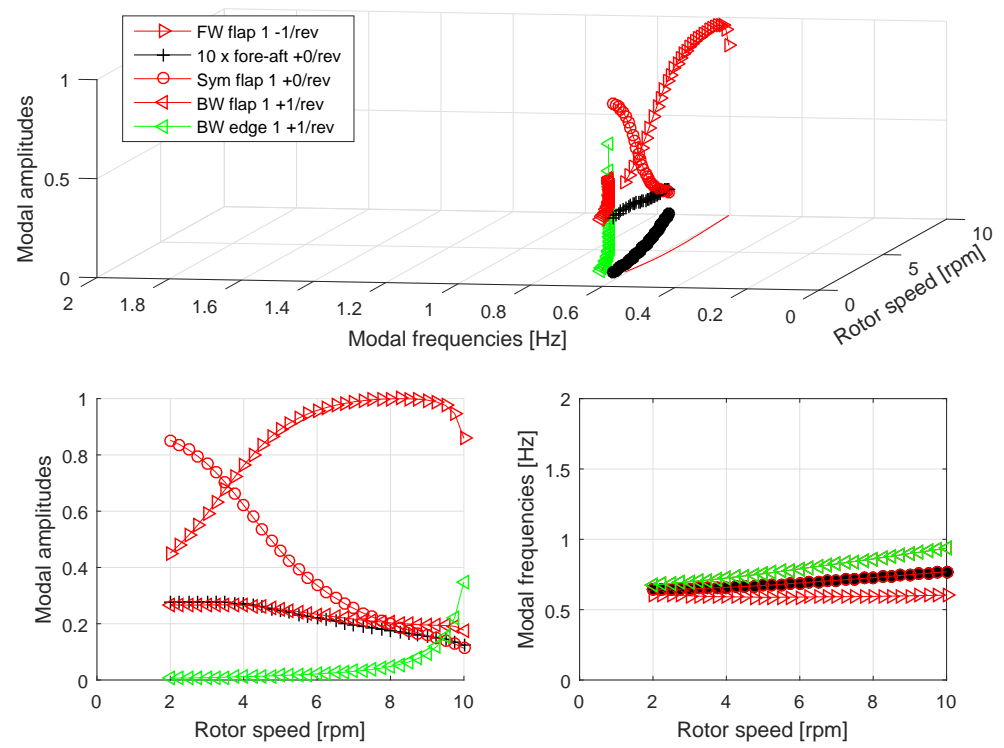

Figure 9. Harmonic modal components for the three-bladed turbine mode dominated by forward whirling (FW) of the rotor in the first flapwise DOF across the rotor speed range. Plot layout as in Fig. 5.
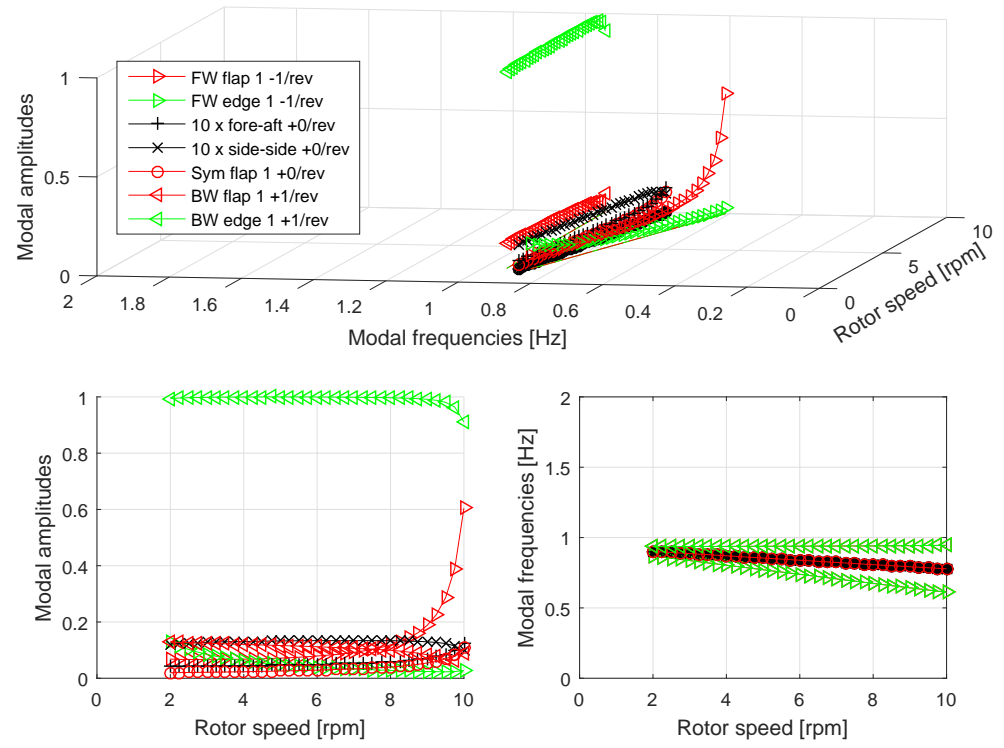

Figure 10. Harmonic modal components for the three-bladed turbine mode dominated by backward whirling (BW) of the rotor in the edgewise DOF. Plot layout as in Fig. 5.

FW components in the first edgewise blade mode and generator rotation due to the nacelle roll. The first BW flapwise mode (Fig. 7) is not pure and also contains symmetric and FW components. The strongly coupled first symmetric (Fig. 8) and FW (Fig. 9) flapwise modes are also not pure and contain other components as well. Note that a BW edgewise component (in green) appears in the first FW flapwise mode at the highest rotor speeds where its BW flapwise component approaches the edgewise blade frequency of about $0.93 \mathrm{~Hz}$. Similarly, the FW flapwise components appear in the first BW edgewise mode (Fig. 10) at higher rotor speeds. The first FW edgewise mode (Fig. 11) contains a small BW edgewise component and a larger FW flapwise component. Similar couplings can be seen for the remaining modes. The second flapwise whirling modes (Figs. 12 and 13) have the largest blade response at a frequency of about $1.5 \mathrm{~Hz}$ (the $+1 /$ rev for BW and $-1 /$ rev for the FW components). The amplitude ratio of these flapwise DOFs are almost constant, indicating that the rotor modes are constructed as a combination of the two blade modes with the isolated modal frequen- 

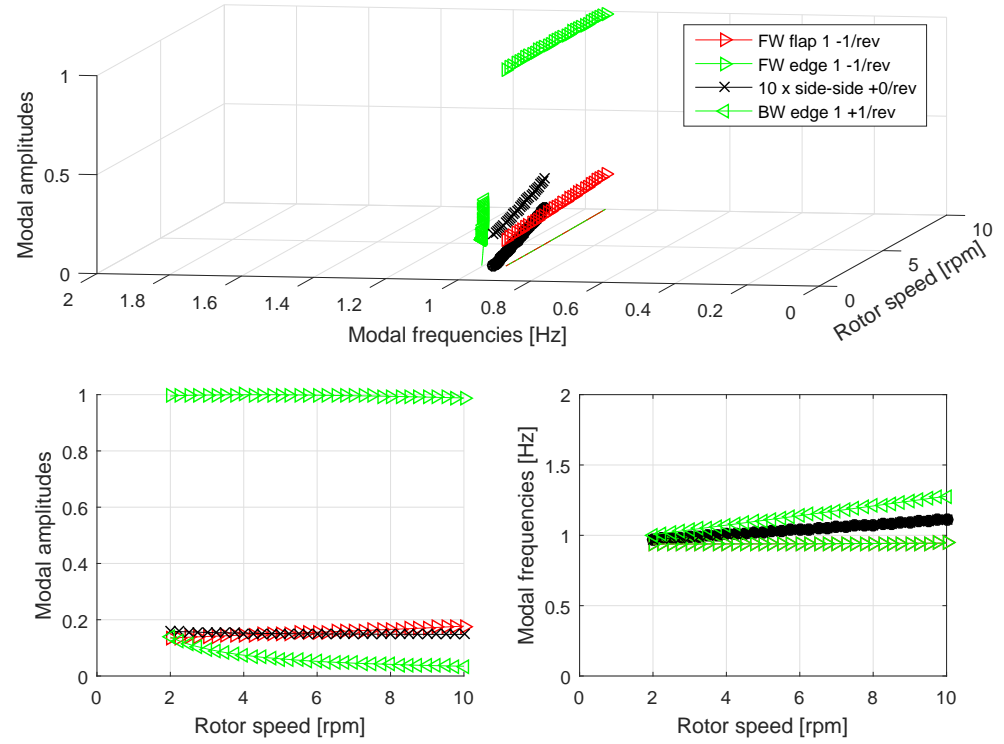

Figure 11. Harmonic modal components for the three-bladed turbine mode dominated by forward whirling (FW) of the rotor in the edgewise DOF. Plot layout as in Fig. 5.
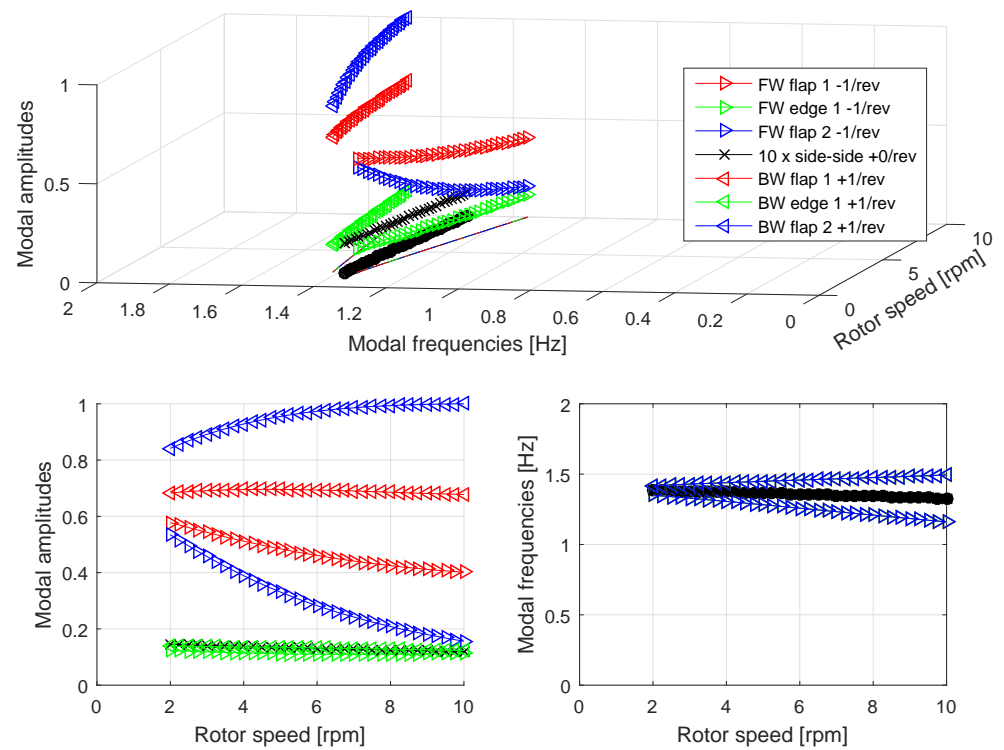

Figure 12. Harmonic modal components for the three-bladed turbine mode dominated by backward whirling (BW) of the rotor in the second flapwise DOF. Plot layout as in Fig. 5.

cies 0.61 and $1.74 \mathrm{~Hz}$. The first symmetric edgewise mode (Fig. 15) couples with both the generator rotation and the shaft torsion, but not with any whirling components. The second symmetric flapwise mode (Fig. 14) contains whirling components that are just below the selected threshold for the plotted amplitudes and therefore not shown.

\subsection{Periodic mode shapes of two-bladed turbines}

Figures 16-23 show the dominant modal components of selected DOFs in the periodic mode shapes of the two-bladed turbine. The scaling of DOF amplitudes, plot layout, and color coding are the same as for the three-bladed turbine. The amplitudes of rotor motion are again computed from the periodic eigenvector using Eq. (39).

A general observation for the two-bladed turbine modes is the larger amplitudes and the higher order of the harmonic components compared to the three-bladed turbine. Another 

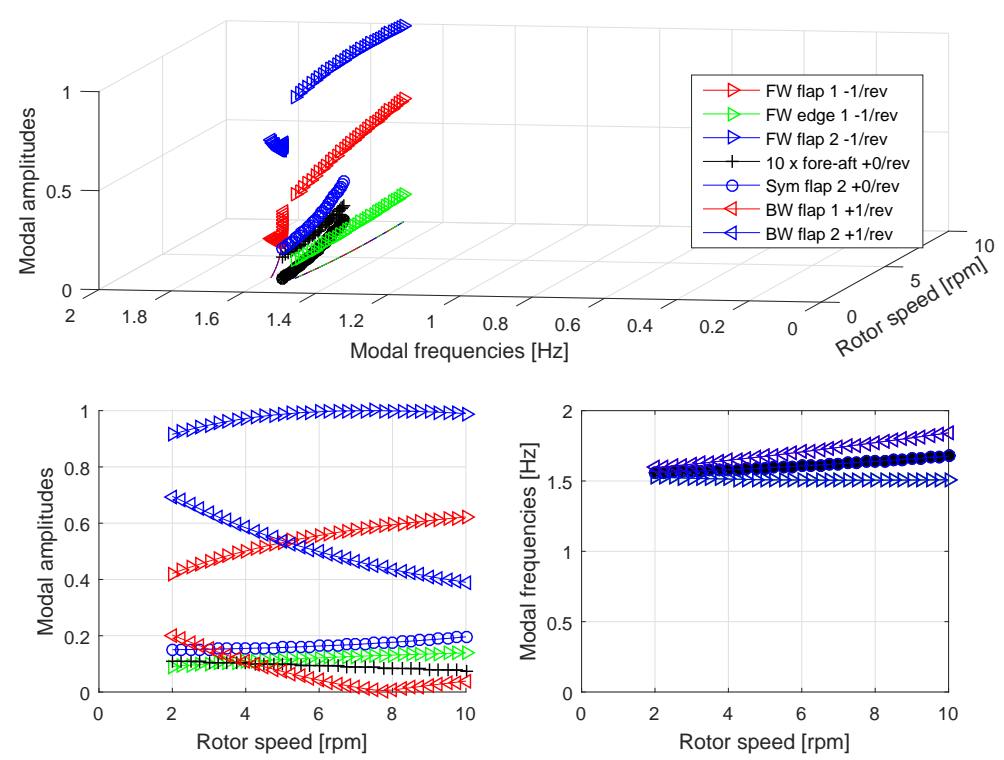

Figure 13. Harmonic modal components for the mode dominated by forward whirling (FW) of the rotor in the second flapwise DOF. Plot layout as in Fig. 5.

observation is that symmetric rotor components always have frequencies that are an even number of 1/rev away from the principal frequency, whereas frequencies for antisymmetric components are shifted an odd number.

Looking at the individual mode shapes, the increased number of harmonics also increases the number of couplings between the different DOFs in each mode. The tower fore-aft mode in Fig. 16 couples again with \pm 1 /rev asymmetric rotor motion as for the three-bladed turbine. However, couplings to higher harmonic components of the blade DOFs are much more dominant at rotor speeds where their frequency crosses the corresponding blade frequency. Such resonant couplings occur for the symmetric $+4 / \mathrm{rev}$ and $+6 / \mathrm{rev}$ components and antisymmetric $+3 / \mathrm{rev}$ and $+5 / \mathrm{rev}$ components of the first flapwise DOF when their frequencies are close to the first flapwise blade frequency, $0.61 \mathrm{~Hz}$, as well as for the antisymmetric $+5 / \mathrm{rev}$ component of the first edgewise DOF when its frequency crosses $0.93 \mathrm{~Hz}$.

The tower side-side mode in Fig. 17 is similar to the same mode of the three-bladed turbine with the coupling to the generator rotation and the $\pm 1 /$ rev harmonics of asymmetric rotor responses.

The symmetric and antisymmetric flapwise modes in Figs. 18 and 19 interchange mode shapes at low rotor speeds; however, they are here named after their dominant components at the higher rotor speeds. The antisymmetric flapwise mode couples with tower fore-aft motion around $6.5 \mathrm{rpm}$, where the frequency of the $-4 / \mathrm{rev}$ component of this DOF is crossing the tower fore-aft frequency of about $0.28 \mathrm{~Hz}$. A similar coupling is seen for the $-6 / \mathrm{rev}$ component of the tower fore-aft DOF in the antisymmetric edgewise mode in Fig. 20 at $8.2 \mathrm{rpm}$. This mode also contains $0 / \mathrm{rev}$ and
$-2 /$ rev components of the tower side-side DOF such that the $\pm 1 /$ rev splitting known from three-bladed rotors appears in the ground-fixed frame.

For the second antisymmetric flapwise mode in Fig. 21, the antisymmetric $+1 /$ rev components of the two flapwise blade DOFs are the two largest components with an almost constant frequency of around $1.46 \mathrm{~Hz}$ (a combination of the two flapwise blade modes as for the three-bladed turbine). The blade motion also contains smaller antisymmetric $-1 / \mathrm{rev}$ and $+3 / \mathrm{rev}$ components of the two flapwise blade DOFs, whose frequencies are decreasing and increasing with $2 / \mathrm{rev}$ in the rotating blade frame. Resonant couplings with symmetric $+6,+8$, and +10 /rev second flapwise DOF and $+6 /$ rev first edgewise DOF are observed when their frequencies are crossing the principal frequencies of the first symmetric edgewise and second symmetric flapwise turbine modes around $1.75 \mathrm{~Hz}$. These two symmetric modes in Figs. 22 and 23 are similar to the corresponding modes of the three-bladed turbine (see Figs. 14 and 15), except that the second symmetric flapwise mode of the two-bladed turbine contains resonant couplings of the antisymmetric $-3 / \mathrm{rev}$ and $-5 /$ rev components of the two flapwise blade DOFs when their frequencies are crossing the frequency of the second antisymmetric flapwise mode (see Fig. 21) around $1.46 \mathrm{~Hz}$.

\subsection{Comparison of modal dynamics of two- and three-bladed turbines}

The modal dynamics of the turbines with two- and threebladed rotors show several similarities but also significant differences: 

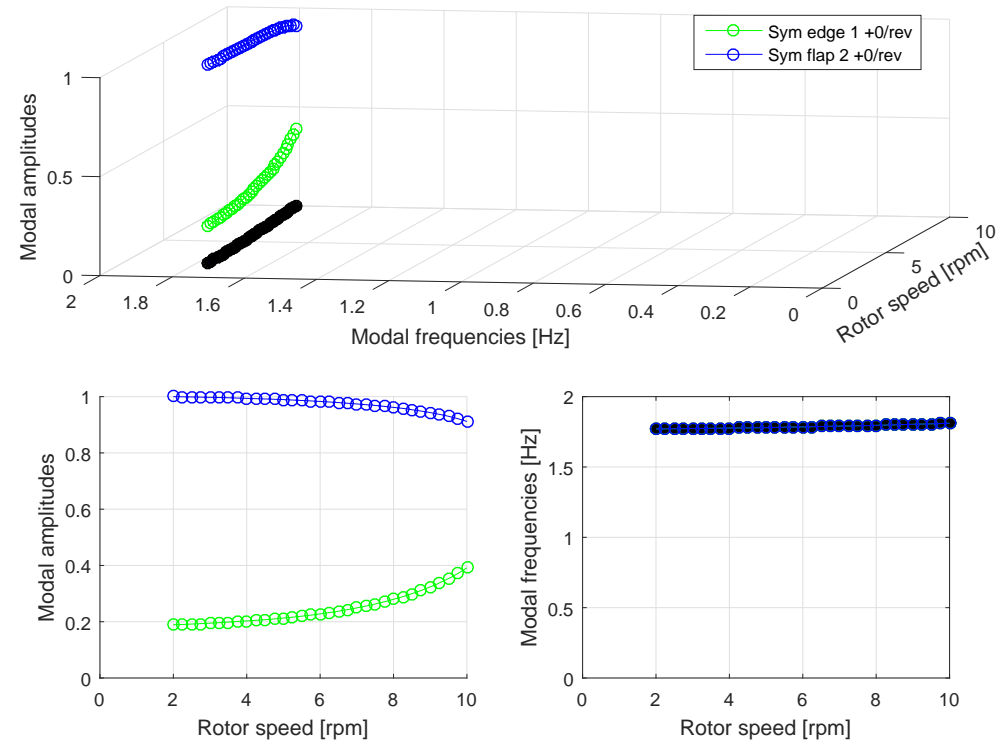

Figure 14. Harmonic modal components for the mode dominated by symmetric deflection of the rotor in the second flapwise mode. Plot layout as in Fig. 5.
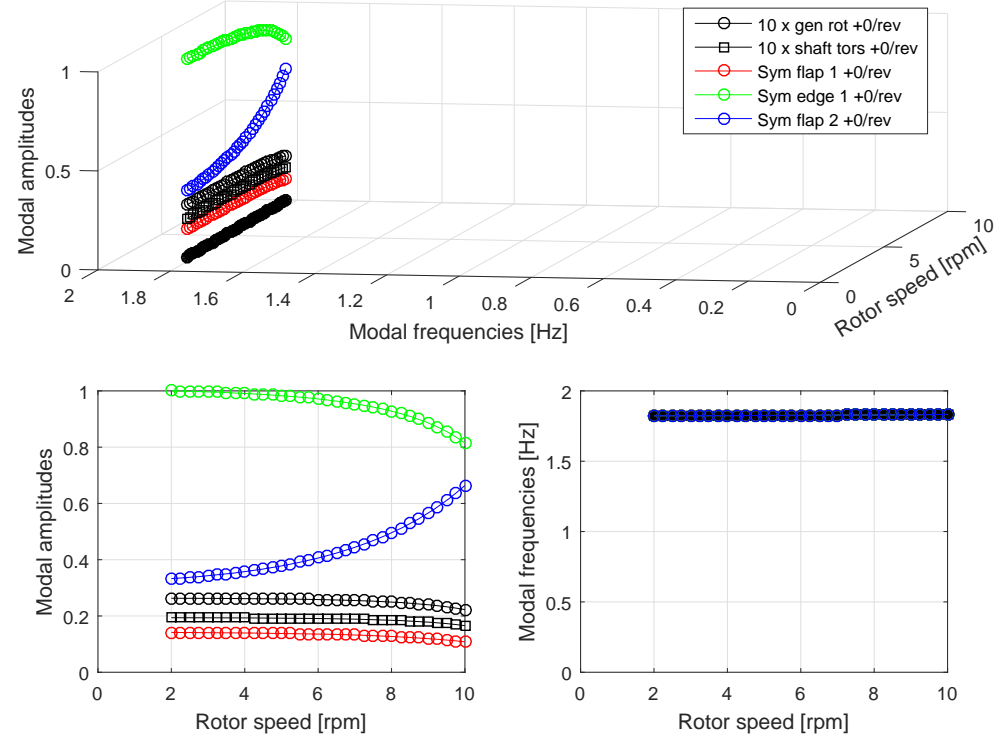

Figure 15. Harmonic modal components for the mode dominated by symmetric deflection of the rotor in the edgewise mode. Plot layout as in Fig. 5.

- The rigid-body drivetrain rotation mode (trivial and therefore not shown) is identical for the two turbines.

- Tower bending modes are similar in frequencies and shapes, except that the fore-aft mode for the two-bladed turbine may contain large components of the first flapwise blade mode when the rotor speed is such that these frequencies of the higher harmonic components are crossing the modal frequency of this blade mode.
- The first symmetric edgewise rotor mode is very similar in frequency and shape because its reaction forces do not couple to other DOFs through large periodic terms in the system matrix.

- The symmetric flapwise rotor modes are similar in frequency and shape, except that the first symmetric flapwise mode of the two-bladed turbine in Fig. 18 has a small -2/rev component of the tower fore-aft DOF, and the second symmetric flapwise mode in Fig. 23 has resonant couplings to antisymmetric flapwise mode. 

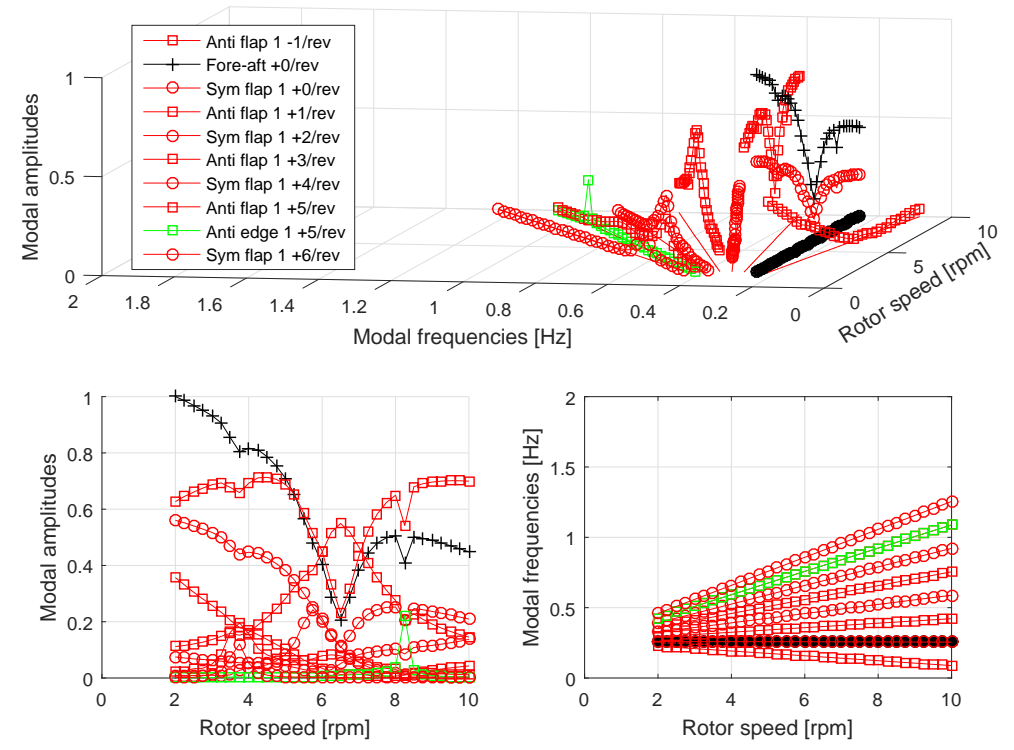

Figure 16. Harmonic modal components for the two-bladed turbine mode dominated by tower fore-aft motion. Plot layout as in Fig. 5.
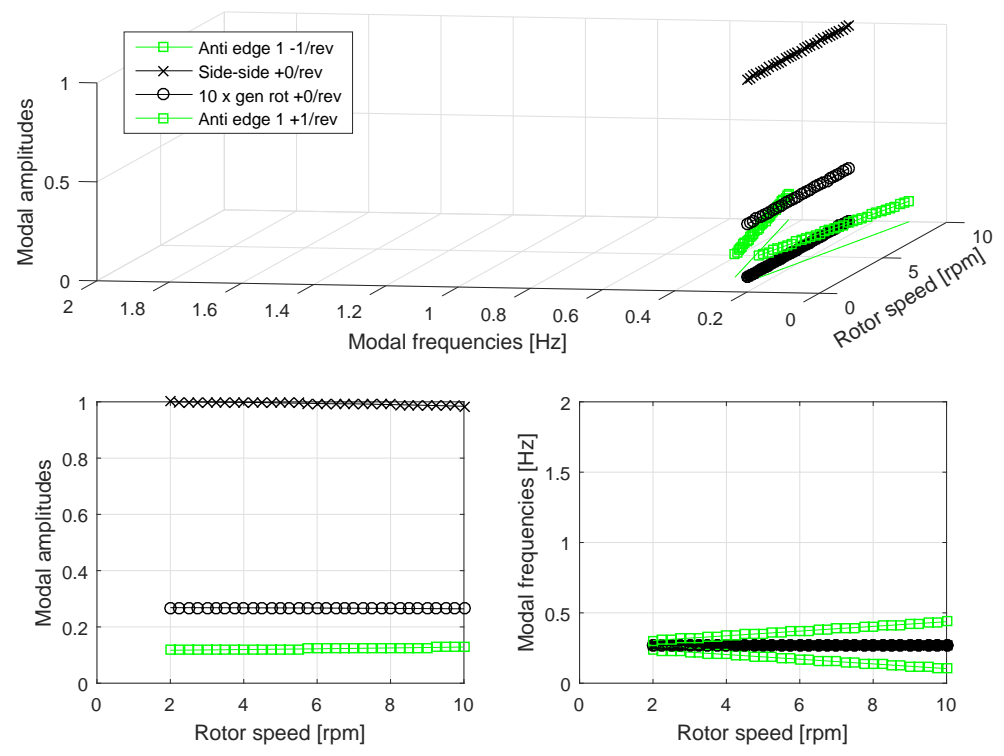

Figure 17. Harmonic modal components for the two-bladed turbine mode dominated by tower side-side motion. Plot layout as in Fig. 5.

- Asymmetric rotor modes: the antisymmetric modes for the two-bladed turbine and the whirling mode pairs of the three-bladed turbine may seem similar when observed from the ground-fixed frame such as a top tower acceleration signal, where the well-known \pm 1 /rev splitting of the frequency peaks is seen. For example, the tower side-side responses at $\pm 1 / \mathrm{rev}$ around the blade edgewise frequency is observed for both the antisymmetric edgewise mode in Fig. 20 and for the edgewise whirling mode pair in Figs. 10 and 11. This similarity has probably caused the misinterpretation of the simulated and measured two-bladed responses in Kim et al. (2015) and Larsen and Kim (2015), but rotor modes of a two-bladed turbine will often have more frequency peaks in both the ground-fixed frame and the rotating blade frame.

The additional modal couplings that exist for two-bladed turbines have the effect that there are additional ways that the modes can be excited by either resonances or interactions with external forces, and that it becomes difficult to interpret frequency spectra from simulations and measurements. To illustrate these effects, the harmonic components of the tower side-side DOF in all modes are plotted in three-dimensional periodic Campbell diagrams for both turbines in Fig. 24. 

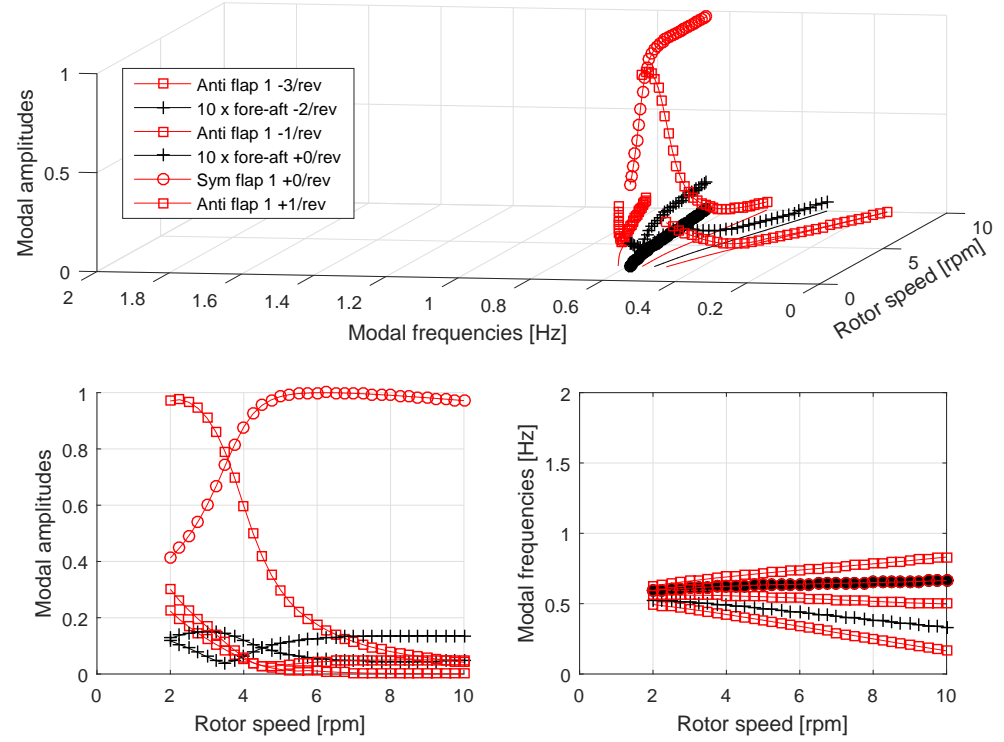

Figure 18. Harmonic modal components for the two-bladed turbine mode dominated by symmetric deflection of the rotor in the first flapwise DOF across the rotor speed range. Plot layout as in Fig. 5.
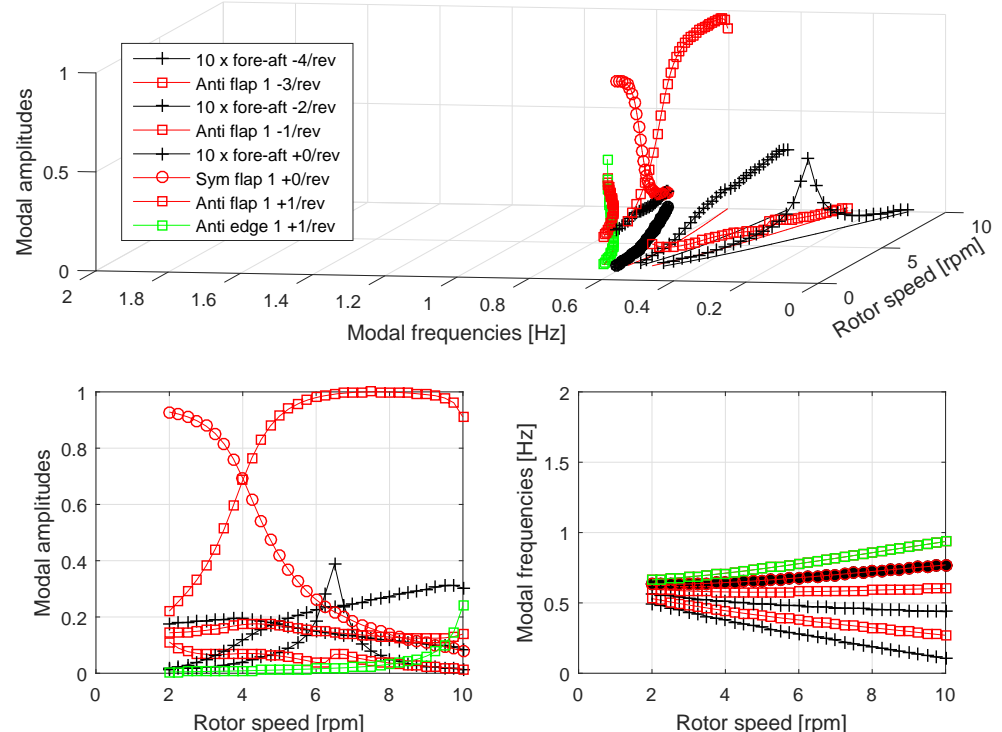

Figure 19. Harmonic modal components for the two-bladed turbine mode dominated by antisymmetric deflection of the rotor in the first flapwise DOF across the rotor speed range. Plot layout as in Fig. 5.

These plots show qualitatively the response in a tower sideside signal when all modes are excited equally. There are clear similarities between the dominant side-side responses for the two turbines. However, where these responses in the ground-fixed frame for the three-bladed turbine only occur on the principal modal frequencies plotted in its conventional Campbell diagram (see Fig. 4), the modes of two-bladed turbine lead to responses in the ground-fixed frame at many other frequencies. The conventional Campbell diagram containing only the principal modal frequencies of a two-bladed turbine therefore has little value, and one should be careful when interpreting frequency response plots for two-bladed turbines.

\section{Conclusions}

The modal dynamics of structures with bladed isotropic rotors (identical and equidistantly spaced blades) have been analyzed using the periodic mode shapes obtained using Hill's method on the linear periodic first-order system equation. 

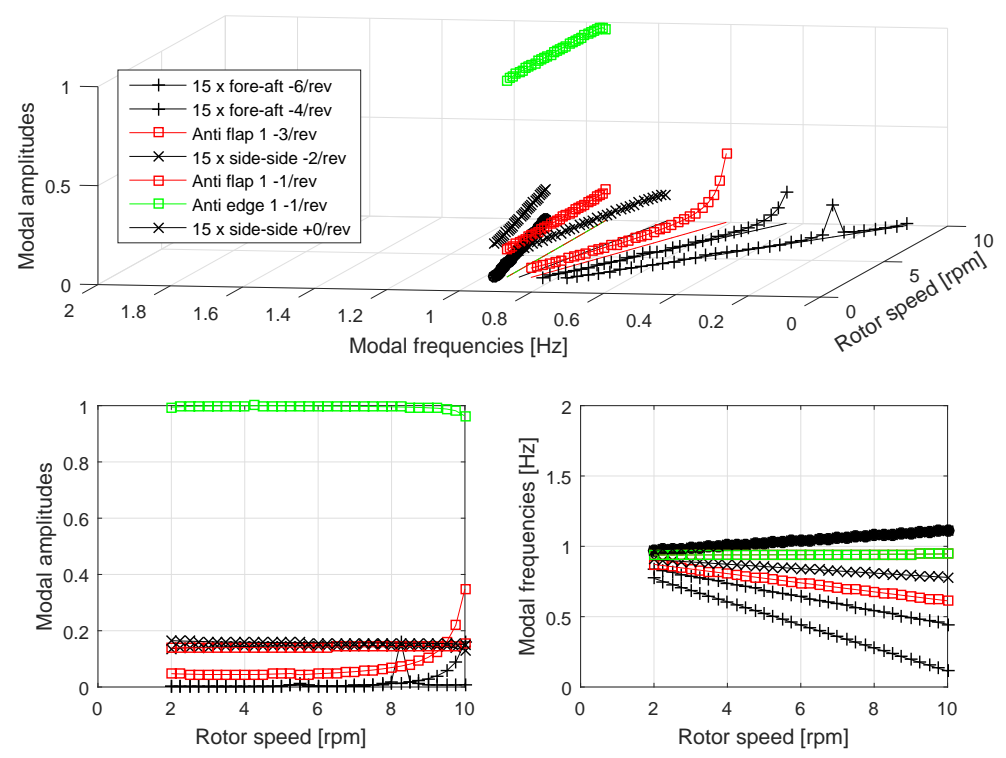

Figure 20. Harmonic modal components for the two-bladed turbine mode dominated by antisymmetric deflection of the rotor in the edgewise DOF. Plot layout as in Fig. 5.

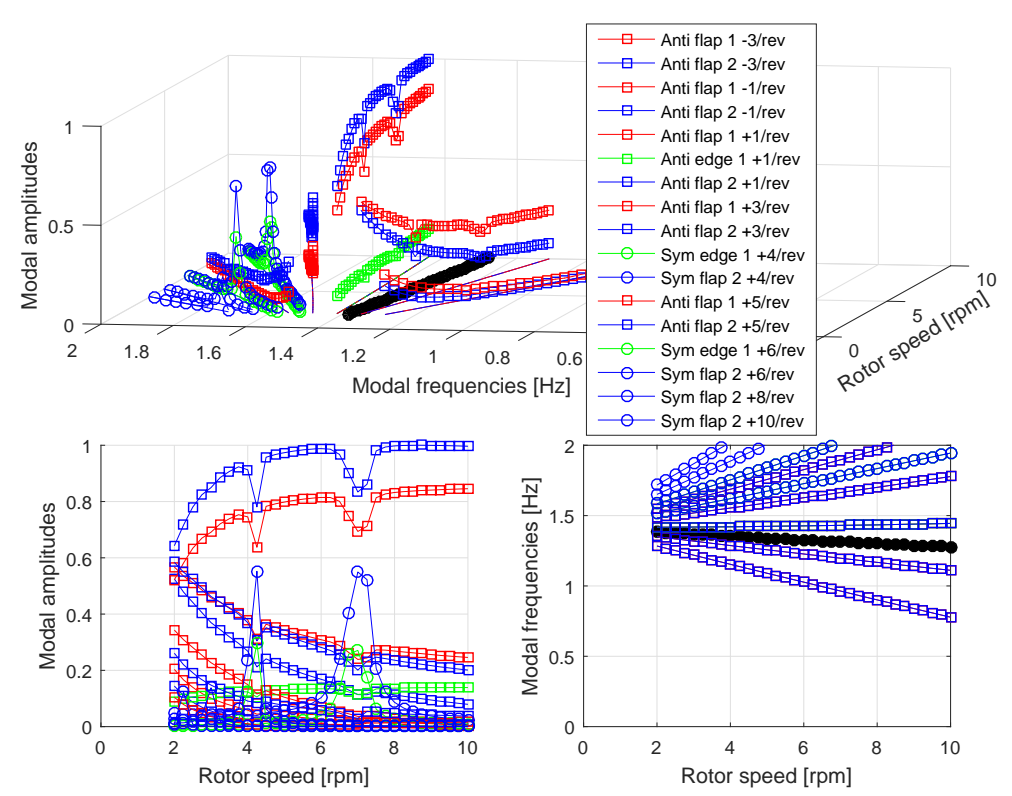

Figure 21. Harmonic modal components for the two-bladed turbine mode dominated by antisymmetric deflection of the rotor in the second flapwise DOF. Plot layout as in Fig. 5.

Analytical derivation of linear second-order equations of motion in a generic form has shown that only $1 / \mathrm{rev}$ harmonics occur in the periodic terms when a rotor has more than two blades, whereas a two-bladed rotor also has $2 /$ rev terms. Analytical inversion of the periodic mass matrix has shown that its highest harmonic term $2 / \mathrm{rev}$ for an isotropic rotor with more than two blades. The inverse mass matrix for a twobladed rotor has been shown to have an infinite Fourier series of component matrices whose norm decreases with the harmonic order. The periodic system matrix of isotropic rotors with more than two blades can therefore be represented by an exact Fourier series, with $3 /$ rev being the highest order, whereas for two-bladed rotors it must be approximated by a truncated Fourier series of predictable accuracy.

Through use of the analytic Fourier series of the system matrix to set up Hill's truncated eigenvalue problem, its principal solutions have been automatically identified by a novel method applicable to larger systems. The symmetric and an- 

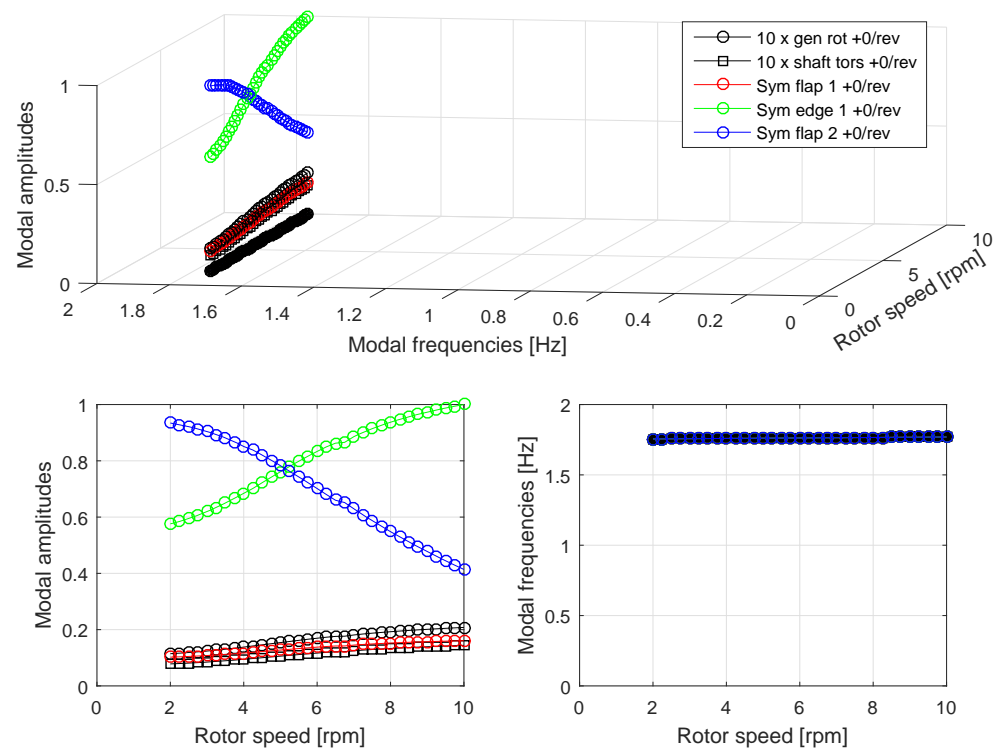

Figure 22. Harmonic modal components for the two-bladed turbine mode dominated by symmetric deflection of the rotor in the edgewise DOF for the higher rotor speeds. Plot layout as in Fig. 5.
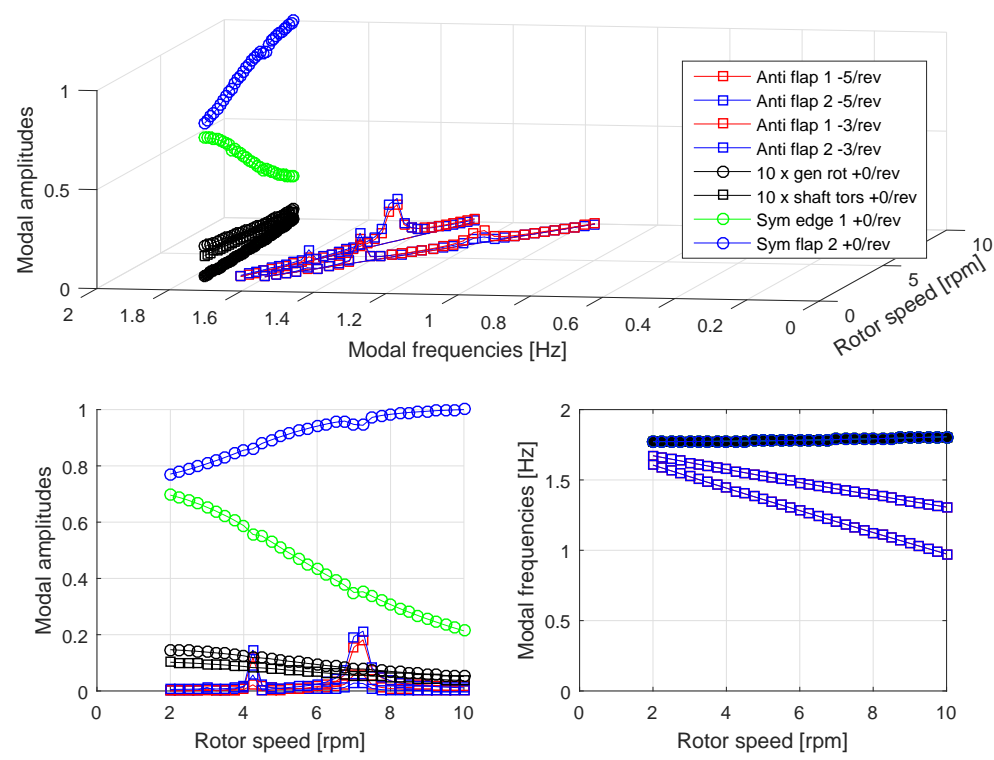

Figure 23. Harmonic modal components for the two-bladed turbine mode dominated by symmetric deflection of the rotor in the second flapwise DOF. Plot layout as in Fig. 5.

tisymmetric modal components of rotors with two blades and the additional whirling components of rotors with more blades have been extracted directly from the periodic eigenvector corresponding to each principal eigen-solution.

As relevant examples, the generic methods have been used to model and analyze the modal dynamics of both two- and three-bladed versions of a $10 \mathrm{MW}$ turbine. The motion of each blade has been described by its three first mode shapes, and the nacelle motion and drivetrain rotations have been described by seven DOFs. Similarities and significant differ- ences between the modal dynamics of the turbines with twoand three-bladed rotors have been found and summarized in Sect. 4.6. A difference is that the larger number and magnitudes of the harmonic components in the system matrix of two-bladed turbines leads to resonant couplings in the mode shapes that have not been observed for the three-bladed turbine. These couplings between DOFs arise when the rotor speed is such that the frequency of a higher harmonic component of one turbine mode is in the vicinity of the frequency of another blade or turbine mode. Another difference is that 

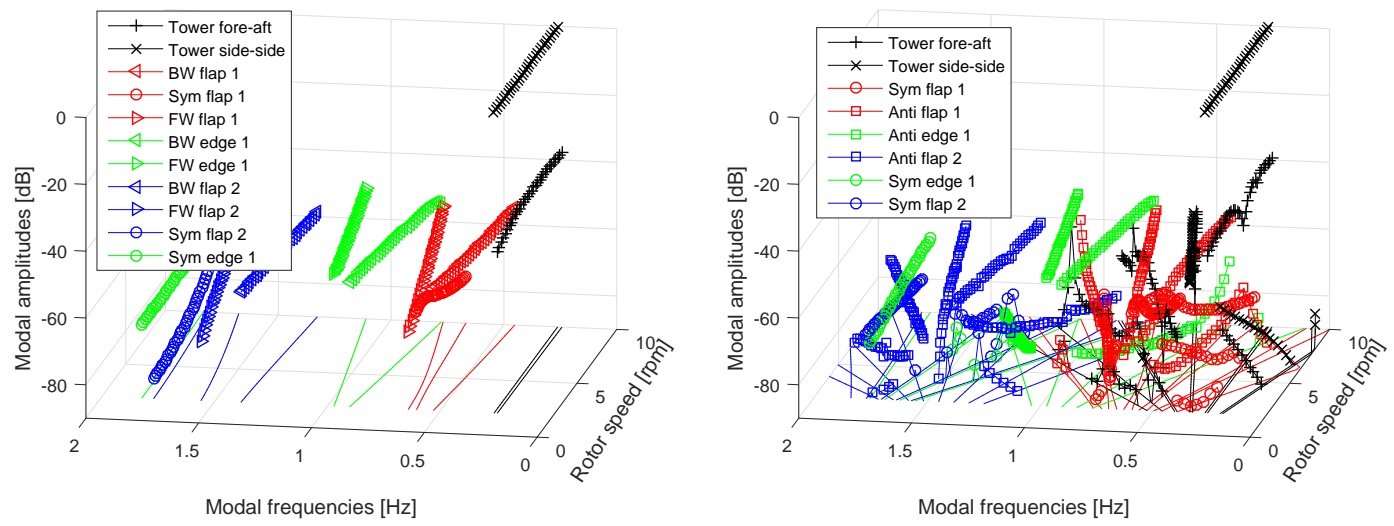

Figure 24. Periodic Campbell diagrams of all harmonic components of the tower side-side DOF in all investigated modes for the threebladed (left panel) and two-bladed (right panel) version of the DTU 10 MW RWT.

a single mode of a two-bladed turbine can lead to responses at several frequencies in both the ground-fixed and rotating blade frames of reference, which complicates the interpretation of simulated or measured turbine responses without the theories presented in this paper.

\section{Data availability}

A repository (Hansen, 2016) contains the model input and Matlab function that generates the system block matrices in Eqs. (13) and (14) for the example with the DTU $10 \mathrm{MW}$ reference wind turbine. Using these matrices and the theory presented in the paper, the reader should be able reproduce the results. 


\section{Appendix A: Elements of system matrices}

This appendix contains a list of the elements of the block matrices in Eqs. (13) and (14) related to the inertia forces and derived by insertion of Eqs. (10), (8), and (9) into the coefficients (Eq. 7), and summation over all volumes of the structure, the ground-fixed substructure $\mathcal{V}_{g}$, and each blade $\mathcal{V}_{\mathrm{b}}$, where several properties of the rotation matrix $\boldsymbol{r}$ and its components have been utilized (see, e.g., Krenk, 2009). The subscripts "g" and "b" are omitted from the DOFs $u_{i}$ and $u_{j}$ for brevity.

The elements of the block matrices for the blade DOFs are

$m_{b, i j}=\int_{\mathcal{V}_{\mathrm{b}}} \rho \frac{\partial \boldsymbol{r}_{\mathrm{b}}^{T}}{\partial u_{i}} \frac{\partial \boldsymbol{r}_{\mathrm{b}}}{\partial u_{j}} d \mathcal{V}_{\mathrm{b}}$

$g_{b, i j}=4 \Omega \int_{\mathcal{V}_{\mathrm{b}}} \rho \frac{\partial \boldsymbol{r}_{\mathrm{b}}^{T}}{\partial u_{i}} \mathbf{B}_{1} \frac{\partial \boldsymbol{r}_{\mathrm{b}}}{\partial u_{j}} d \mathcal{V}_{\mathrm{b}}$

$s_{b, i j}=-\Omega^{2} \int_{\mathcal{V}_{\mathrm{b}}} \rho\left(\frac{\partial^{2} \boldsymbol{r}_{\mathrm{b}}^{T}}{\partial u_{i} \partial u_{j}} \mathbf{A}_{1} \boldsymbol{r}_{\mathrm{b}}+\frac{\partial \boldsymbol{r}_{\mathrm{b}}^{T}}{\partial u_{i}} \mathbf{A}_{1} \frac{\partial \boldsymbol{r}_{\mathrm{b}}}{\partial u_{j}}\right) d \mathcal{V}_{\mathrm{b}}$,

where $\mathbf{A}_{1}=\frac{1}{2}\left(\mathbf{R}_{1}-\overline{\mathbf{R}}_{1}\right)$ and $\mathbf{B}_{1}=\frac{1}{2}\left(\mathbf{R}_{1}+\overline{\mathbf{R}}_{1}\right)$ are the symmetric real part and the antisymmetric imaginary part of the matrix $\mathbf{R}_{1}$. Note that $\mathbf{R}_{0}^{T}=\mathbf{R}_{0}$ and $\overline{\mathbf{R}}_{1}=\mathbf{R}_{1}^{T}$. The elements of the block matrices for the DOFs of the ground-fixed substructure are

$$
\begin{aligned}
m_{g, 0, i j} & =\int_{\mathcal{V}_{\mathrm{g}}} \rho \frac{\partial \boldsymbol{r}_{\mathrm{g}}^{T}}{\partial u_{i}} \frac{\partial \boldsymbol{r}_{\mathrm{g}}}{\partial u_{j}} d \mathcal{V}_{\mathrm{g}}+B \int_{\mathcal{V}_{\mathrm{b}}} \rho\left(\frac{\partial \boldsymbol{r}_{\mathrm{c}}^{T}}{\partial u_{i}} \frac{\partial \boldsymbol{r}_{\mathrm{c}}}{\partial u_{j}}\right. \\
& +\boldsymbol{r}_{\mathrm{b}}^{T} \mathbf{R}_{0}\left(\frac{\partial \mathbf{T}_{\mathrm{c}}^{T}}{\partial u_{i}} \frac{\partial \boldsymbol{r}_{\mathrm{c}}}{\partial u_{j}}+\frac{\partial \mathbf{T}_{\mathrm{c}}^{T}}{\partial u_{j}} \frac{\partial \boldsymbol{r}_{\mathrm{c}}}{\partial u_{i}}\right)+\boldsymbol{r}_{\mathrm{b}}^{T}\left(\mathbf{R}_{0} \frac{\partial \mathbf{T}_{\mathrm{c}}^{T}}{\partial u_{i}} \frac{\partial \mathbf{T}_{\mathrm{c}}}{\partial u_{j}} \mathbf{R}_{0}\right. \\
& \left.\left.+\mathbf{R}_{1}\left(\frac{\partial \mathbf{T}_{\mathrm{c}}^{T}}{\partial u_{i}} \frac{\partial \mathbf{T}_{\mathrm{c}}}{\partial u_{j}}+\frac{\partial \mathbf{T}_{\mathrm{c}}^{T}}{\partial u_{j}} \frac{\partial \mathbf{T}_{\mathrm{c}}}{\partial u_{i}}\right) \mathbf{R}_{1}\right) \boldsymbol{r}_{\mathrm{b}}\right) d \mathcal{V}_{\mathrm{b}} \\
m_{g, 2, i j}= & \left\{\begin{array}{c}
2 \int_{\mathcal{V}_{\mathrm{b}}} \rho \boldsymbol{r}_{\mathrm{b}}^{T} \mathbf{R}_{1}^{T} \frac{\partial \mathbf{T}_{\mathrm{c}}^{T}}{\partial u_{i}} \frac{\partial \mathbf{T}_{\mathrm{c}}}{\partial u_{j}} \mathbf{R}_{1} \boldsymbol{r}_{\mathrm{b}} d \mathcal{V}_{\mathrm{b}} \quad \text { for } B=2 \\
0 \quad \text { otherwise }
\end{array}\right. \\
g_{g, 0, i j}= & \iota 2 \Omega B \int_{\mathcal{V}_{\mathrm{b}}} \rho \boldsymbol{r}_{\mathrm{b}}^{T} \mathbf{R}_{1}\left(\frac{\partial \mathbf{T}_{\mathrm{c}}^{T}}{\partial u_{i}} \frac{\partial \mathbf{T}_{\mathrm{c}}}{\partial u_{j}}-\frac{\partial \mathbf{T}_{\mathrm{c}}^{T}}{\partial u_{j}} \frac{\partial \mathbf{T}_{\mathrm{c}}}{\partial u_{i}}\right) \mathbf{R}_{1} \boldsymbol{r}_{\mathrm{b}} d \mathcal{V}_{\mathrm{b}} \\
g_{g, 2, i j}= & \begin{array}{c}
\iota \Omega \int_{\mathcal{V}_{\mathrm{b}}} \rho \boldsymbol{r}_{\mathrm{b}}^{T} \mathbf{R}_{1}^{T} \frac{\partial \mathbf{T}_{\mathrm{c}}^{T}}{\partial u_{i}} \frac{\partial \mathbf{T}_{\mathrm{c}}}{\partial u_{j}} \mathbf{R}_{1} \boldsymbol{r}_{\mathrm{b}} d \mathcal{V}_{\mathrm{b}} \quad \text { for } B=2 \\
0 \quad \text { otherwise }
\end{array} \quad(\mathrm{A} 2)
\end{aligned}
$$

The elements of the block matrices that couple the blade and ground-fixed DOFs are

$$
\begin{aligned}
m_{g b, 0, i j} & =\int_{\mathcal{V}_{\mathrm{b}}} \rho\left(\frac { \partial \boldsymbol { r } _ { \mathrm { c } } ^ { T } } { \partial u _ { i } } \mathbf { T } _ { \mathrm { c } } \mathbf { R } _ { 0 } \frac { \partial \boldsymbol { r } _ { \mathrm { b } } } { \partial u _ { j } } \boldsymbol { r } _ { \mathrm { b } } ^ { T } \left(\mathbf{R}_{0} \frac{\partial \mathbf{T}_{\mathrm{c}}^{T}}{\partial u_{i}} \mathbf{T}_{\mathrm{c}} \mathbf{R}_{0}\right.\right. \\
& \left.\left.+\mathbf{R}_{1}^{T} \frac{\partial \mathbf{T}_{\mathrm{c}}^{T}}{\partial u_{i}} \mathbf{T}_{\mathrm{c}} \mathbf{R}_{1}^{T}+\mathbf{R}_{1} \frac{\partial \mathbf{T}_{\mathrm{c}}^{T}}{\partial u_{i}} \mathbf{T}_{\mathrm{c}} \mathbf{R}_{1}\right) \frac{\partial \boldsymbol{r}_{\mathrm{b}}}{\partial u_{j}}\right) d \mathcal{V}_{\mathrm{b}}
\end{aligned}
$$

$$
\begin{aligned}
& m_{g b, 1, i j}=\int_{\mathcal{V}_{\mathrm{b}}} \rho( \\
& \ldots+\mathbf{R}_{1}^{T} \frac{\partial \mathbf{T}_{\mathrm{c}}^{T}}{\partial u_{i}} \mathbf{T}_{\mathrm{c}} \mathbf{R}_{0} \frac{\partial \boldsymbol{r}_{\mathrm{b}}}{\partial u_{j}} d \mathcal{V}_{\mathrm{b}} \\
& g_{g b, 1, i j}=\imath 2 \Omega \int_{\mathcal{V}_{\mathrm{b}}} \rho\left(\frac{\partial \boldsymbol{r}_{\mathrm{c}}^{T}}{\partial u_{i}} \mathbf{T}_{\mathrm{c}} \mathbf{R}_{1}+\boldsymbol{r}_{\mathrm{b}}^{T} \mathbf{R}_{0} \frac{\partial \mathbf{T}_{\mathrm{c}}^{T}}{\partial u_{i}} \mathbf{T}_{\mathrm{c}} \mathbf{R}_{1}\right) \\
& \frac{\partial \boldsymbol{r}_{\mathrm{b}}}{\partial u_{j}} d \mathcal{V}_{\mathrm{b}} \\
& g_{b g, 0, i j}=\iota 2 \Omega \int_{\mathcal{V}_{\mathrm{b}}} \rho\left(\boldsymbol{r}_{\mathrm{b}}^{T} \mathbf{R}_{1}^{T} \frac{\partial \mathbf{T}_{\mathrm{c}}^{T}}{\partial u_{j}} \mathbf{T}_{\mathrm{c}} \mathbf{R}_{1} \frac{\partial \boldsymbol{r}_{\mathrm{b}}}{\partial u_{i}}-\boldsymbol{r}_{\mathrm{b}}^{T} \mathbf{R}_{1} \frac{\partial \mathbf{T}_{\mathrm{c}}^{T}}{\partial u_{j}}\right. \\
& \left.\mathbf{T}_{\mathrm{c}} \mathbf{R}_{1} \frac{\partial \boldsymbol{r}_{\mathrm{b}}}{\partial u_{\dot{j}}}\right) d \mathcal{V}_{\mathrm{b}} \\
& g_{b g, 1, i j}=\imath 2 \Omega \int_{\mathcal{V}_{\mathrm{b}}} \rho \frac{\partial u_{\mathrm{b}}^{T}}{\partial u_{i}} \mathbf{R}_{0} \mathbf{T}_{\mathrm{c}}^{T} \frac{\partial \mathbf{T}_{\mathrm{c}}}{\partial u_{j}} \mathbf{R}_{1} \boldsymbol{r}_{\mathrm{b}} d \mathcal{V}_{\mathrm{b}} \\
& s_{g b, 1, i j}=\Omega^{2} \int_{\mathcal{V}_{\mathrm{b}}} \rho\left(\boldsymbol{r}_{\mathrm{b}}^{T} \mathbf{R}_{1}^{T} \frac{\partial \mathbf{T}_{\mathrm{c}}^{T}}{\partial u_{i}} \mathbf{T}_{\mathrm{c}} \mathbf{R}_{0}-\frac{\partial \boldsymbol{r}_{\mathrm{c}}^{T}}{\partial u_{i}} \mathbf{T}_{\mathrm{c}} \mathbf{R}_{1}\right. \\
& \left.-\boldsymbol{r}_{\mathrm{b}}^{T} \mathbf{R}_{0} \frac{\partial \mathbf{T}_{\mathrm{c}}^{T}}{\partial u_{i}} \mathbf{T}_{\mathrm{c}} \mathbf{R}_{1}\right) \frac{\partial \boldsymbol{r}_{\mathrm{b}}}{\partial u_{j}} d \mathcal{V}_{\mathrm{b}}
\end{aligned}
$$

\section{Appendix B: Components of inverted mass matrix}

The mean and harmonic components of the block matrices Eq. (28) of the inverse mass matrix Eq. (15) can be computed as

$$
\begin{aligned}
\mathbf{E}_{0}=\mathbf{M}_{\mathrm{r}}^{-1}+\mathbf{M}_{\mathrm{r}}^{-1}\left(\mathbf{M}_{\mathrm{gr}, 1}^{H} \mathbf{H}_{2} \overline{\mathbf{M}}_{\mathrm{gr}, 1}\right. \\
+\mathbf{M}_{\mathrm{gr}, 0}^{T} \mathbf{H}_{0} \mathbf{M}_{\mathrm{gr}, 0}+\mathbf{M}_{\mathrm{gr}, 1}^{H} \mathbf{H}_{0} \mathbf{M}_{\mathrm{gr}, 1}+\mathbf{M}_{\mathrm{gr}, 1}^{T} \mathbf{H}_{0} \overline{\mathbf{M}}_{\mathrm{gr}, 1} \\
\left.\quad+\mathbf{M}_{\mathrm{gr}, 1}^{T} \overline{\mathbf{H}}_{2} \mathbf{M}_{\mathrm{gr}, 1}\right) \mathbf{M}_{\mathrm{r}}^{-1} \\
\mathbf{E}_{2 m+1}=\mathbf{M}_{\mathrm{r}}^{-1}\left(\mathbf{M}_{\mathrm{gr}, 0}^{T} \mathbf{H}_{2 m+2} \overline{\mathbf{M}}_{\mathrm{gr}, 1}+\mathbf{M}_{\mathrm{gr}, 1}^{H} \mathbf{H}_{2 m+2} \mathbf{M}_{\mathrm{gr}, 0}\right. \\
\left.\quad+\mathbf{M}_{\mathrm{gr}, 0}^{T} \mathbf{H}_{2 m} \mathbf{M}_{\mathrm{gr}, 1}+\mathbf{M}_{\mathrm{gr}, 1}^{T} \mathbf{H}_{2 m} \mathbf{M}_{\mathrm{gr}, 0}\right) \mathbf{M}_{\mathrm{r}}^{-1} \\
\mathbf{E}_{2 m+2}=\mathbf{M}_{\mathrm{r}}^{-1}\left(\mathbf{M}_{\mathrm{gr}, 1}^{H} \mathbf{H}_{2 m+4} \overline{\mathbf{M}}_{\mathrm{gr}, 1}\right. \\
\quad+\mathbf{M}_{\mathrm{gr}, 0}^{T} \mathbf{H}_{2 m+2} \mathbf{M}_{\mathrm{gr}, 0}+\mathbf{M}_{\mathrm{gr}, 1}^{H} \mathbf{H}_{2 m+2} \mathbf{M}_{\mathrm{gr}, 1} \\
\left.\quad+\mathbf{M}_{\mathrm{gr}, 1}^{T} \mathbf{H}_{2 m+2} \overline{\mathbf{M}}_{\mathrm{gr}, 1}+\mathbf{M}_{\mathrm{gr}, 1}^{T} \mathbf{H}_{2 m} \mathbf{M}_{\mathrm{gr}, 1}\right) \mathbf{M}_{\mathrm{r}}^{-1}
\end{aligned}
$$

and

$$
\begin{aligned}
& \mathbf{F}_{2 m}=-\mathbf{H}_{2 m} \mathbf{M}_{\mathrm{gr}, 0} \mathbf{M}_{\mathrm{r}}^{-1} \\
& \mathbf{F}_{2 m+1}=-\mathbf{H}_{2 m+2} \overline{\mathbf{M}}_{\mathrm{gr}, 1} \mathbf{M}_{\mathrm{r}}^{-1}-\mathbf{H}_{2 m} \mathbf{M}_{\mathrm{gr}, 1} \mathbf{M}_{\mathrm{r}}^{-1},
\end{aligned}
$$

where $m=0,1, \ldots, N_{\mathrm{h}},(\cdot)^{H}$ denotes the conjugate transpose matrix operator, the mean and harmonic components of the block matrix $\mathbf{H}$ Eq. (20) can be found in Eqs. (22) and (23), and the conditions $\mathbf{H}_{2 m}=\mathbf{0}$ for $m>N_{\mathrm{h}}$ must be used. Note that $\mathbf{E}_{-n}=\overline{\mathbf{E}}_{n}$ and $\mathbf{F}_{-n}=\overline{\mathbf{F}}_{n}$. 


\section{Appendix C: Components of system matrix}

The components of the system matrix Eq. (29) can be written as

$$
\mathbf{A}_{n}=\left[\begin{array}{cccc}
\multicolumn{2}{c}{\mathbf{0}} & \multicolumn{2}{c}{\mathbf{A}_{12}} \\
\mathbf{A}_{21,11, n} & \mathbf{A}_{21,12, n} & \mathbf{A}_{22,11, n} & \mathbf{A}_{22,12, n} \\
\mathbf{A}_{21,21, n} & \mathbf{A}_{21,22, n} & \mathbf{A}_{22,21, n} & \mathbf{A}_{22,22, n}
\end{array}\right],
$$

where $\mathbf{A}_{12}=\mathbf{I}$ for $n=0$ and $\mathbf{A}_{12}=\mathbf{0}$ for $n>0$, and the block matrices can be computed as

$\mathbf{A}_{21,11, n}=-\mathbf{E}_{n}\left(\mathbf{K}_{\mathrm{r}}+\mathbf{S}_{\mathrm{r}}\right)-\mathbf{F}_{n-1}^{T} \mathbf{S}_{\mathrm{gr}, 1}-\mathbf{F}_{n+1}^{T} \overline{\mathbf{S}}_{\mathrm{gr}, 1}$

$\mathbf{A}_{21,21, n}=-\mathbf{F}_{n}\left(\mathbf{K}_{\mathrm{r}}+\mathbf{S}_{\mathrm{r}}\right)-\mathbf{H}_{n-1}^{T} \mathbf{S}_{\mathrm{gr}, 1}-\mathbf{H}_{n+1}^{T} \overline{\mathbf{S}}_{\mathrm{gr}, 1}$

$\mathbf{A}_{21,12, n}=-\mathbf{F}_{n}^{T} \mathbf{K}_{\mathrm{g}}$

$\mathbf{A}_{21,22, n}=-\mathbf{H}_{n} \mathbf{K}_{\mathrm{g}}$

$\mathbf{A}_{22,11, n}=-\mathbf{E}_{n} \mathbf{C}_{\mathrm{r}}-\mathbf{F}_{n}^{T} \mathbf{G}_{\mathrm{gr}, 0}-\mathbf{F}_{n-1}^{T} \mathbf{G}_{\mathrm{gr}, 1}-\mathbf{F}_{n+1}^{T} \overline{\mathbf{G}}_{\mathrm{gr}, 1}$

$\mathbf{A}_{22,21, n}=-\mathbf{F}_{n} \mathbf{C}_{\mathrm{r}}-\mathbf{H}_{n} \mathbf{G}_{\mathrm{gr}, 0}-\mathbf{H}_{n-1} \mathbf{G}_{\mathrm{gr}, 1}-\mathbf{H}_{n+1} \overline{\mathbf{G}}_{\mathrm{gr}, 1}$

$\mathbf{A}_{22,12, n}=-\mathbf{F}_{n}^{T}\left(\mathbf{G}_{\mathrm{g}, 0}+\mathbf{C}_{\mathrm{g}}\right)-\mathbf{E}_{n-1} \mathbf{G}_{\mathrm{rg}, 1}-\mathbf{E}_{n+1} \overline{\mathbf{G}}_{\mathrm{rg}, 1}$

$$
-\mathbf{F}_{n-2}^{T} \mathbf{G}_{\mathrm{g}, 2}-\mathbf{F}_{n+2}^{T} \overline{\mathbf{G}}_{\mathrm{g}, 2}
$$

$\mathbf{A}_{22,22, n}=-\mathbf{H}_{n}\left(\mathbf{G}_{\mathrm{g}, 0}+\mathbf{C}_{\mathrm{g}}\right)-\mathbf{F}_{n-1} \mathbf{G}_{\mathrm{rg}, 1}-\mathbf{F}_{n+1} \overline{\mathbf{G}}_{\mathrm{rg}, 1}$

$$
-\mathbf{H}_{n-2} \mathbf{G}_{\mathrm{g}, 2}-\mathbf{H}_{n+2} \overline{\mathbf{G}}_{\mathrm{g}, 2}
$$

for $n=0,1, \ldots, 2 N_{\mathrm{h}}+3$ using the following properties of the matrices $\mathbf{E}_{n}, \mathbf{F}_{n}$, and $\mathbf{H}_{n}$ :

$$
\begin{aligned}
& \mathbf{E}_{-n}=\overline{\mathbf{E}}_{n}, \mathbf{E}_{-n}=\overline{\mathbf{E}}_{n}, \mathbf{H}_{-n}=\overline{\mathbf{H}}_{n}, \text { and } \\
& \mathbf{E}_{n+2}=\mathbf{F}_{n+1}=\mathbf{H}_{n}=\mathbf{0} \text { for } n>2 N_{\mathrm{h}} .
\end{aligned}
$$

Note that $N_{\mathrm{h}}=0$ for isotropic rotors with more than two blades, whereas $N_{\mathrm{h}}$ is selected for two-bladed rotors based on the required accuracy of the system matrix. 
Acknowledgements. The support by the Danish Energy Agency through the EUDP-2011 II project "Demonstration of Partial Pitch 2-Bladed Wind Turbine Performance" is gratefully acknowledged. The author would also like to thank Anders M. Hansen, Ilmar F. Santos, Torben J. Larsen, Riccardo Riva, and project partners at Envision Energy for valuable discussions.

Edited by: A. Kolios

Reviewed by: V. A. Riziotis and D. Ossmann

\section{References}

Allemang, R. J.: The Modal Assurance Criterion - Twenty years of use and abuse, Sound Vibrat., 37, 14-21, 2003.

Bak, C., Bitsche, R., Yde, A., Kim, T., Hansen, M. H., Zahle, F., Gaunaa, M., Blasques, J. P. A. A., Døssing, M., Wedel Heinen, J. J., and Behrens, T.: Light Rotor: The 10-MW reference wind turbine, European Wind Energy Association Conference and Exhibition, 16-19 April 2012, Copenhagen, Denmark, 2012.

Bergami, L. and, Hansen, M. H.: High-fidelity linear time-invariant model of a smart rotor with adaptive trailing edge flaps, Wind Energy., doi:10.1002/we.2014, in press, 2016.

Bergami, L., Madsen, H. A., and Rasmussen, F.: A Two-Bladed Teetering Hub configuration for the DTU 10 MW RWT: loads considerations, European Wind Energy Association Conference and Exhibition, 11-13 March 2014, Barcelona, Spain, 2014.

Bir, G.: Multi-blade coordinate transformation and its application to wind turbine analysis, ASME Wind Energy Symposium, January 2008, Reno, USA, 1-15, 2008.

Bottasso, C. and Cacciola, S.: Model-independent periodic stability analysis of wind turbines, Wind Energy, 18, 865-887, doi:10.1002/we.1735, 2015.

Christensen, R. H. and Santos, I. F.:Design of active controlled rotor-blade systems based on time-variant modal analysis, J. Vibrat. Control, 11, 801-828, 2005.

Clough, R. W. and Penzien, J.: Dynamics of Structures, McGrawHill, New Yori, USA, 1975.

Coleman, R. P. and Feingold, A. M.: Theory of self-excited mechanical oscillations of helicopter rotors with hinged blades, Technical Report NACA-TN-3844, NACA-TR-1351, Langley Research Center, Langley Field, VA, USA, 1958.

Curtis, C. W.: On the convergence of Hill's method, Math. Comput., 79, 169-187, 2010.

Ertz, M., Reister, A., and Nordmann, R.: Zur Berechnung der Eigenschwingungen von Strukturen mit periodisch zeitvarianten Bewegungsgleichungen, in: Schwingungen in rotierendenMaschinen (SIRM) III, Vieweg-Verlag, Braunschweig, Wiesbaden, 288-296, 1995.

Golub, G. H. and Van Loan, C. F.: Matrix Computations, 3rd Edn., The Johns Hopkins University Press, Baltimore, MD, 1996.

Hansen, M. H.: Aeroelasticity and Dynamics of Spinning Disks, PhD Thesis, Technical University of Denmark, Lyngby, Denmark, 1999.

Hansen, M. H.: Improved Modal Dynamics of Wind Turbines to Avoid Stall-Induced Vibrations, Wind Energy, 6, 179-195, 2003.

Hansen, M. H.: Aeroelastic Stability Analysis of Wind Turbines Using an Eigenvalue Approach, Wind Energy, 7, 133-143, 2004.

Hansen, M. H.: Aeroelastic Instability Problems for Wind Turbines, Wind Energy, 10, 551-577, 2007.
Hansen, M. H.: Data for Wind Energy Science paper "Modal dynamics of structures with bladed isotropic rotors and its complexity for 2-bladed rotors", doi:10.5281/zenodo.168442, 2016.

Hill, G. W.: On the part of the motion of the lunar perigee which is a function of the mean motions of the sun and moon, Acta Mathematica, 8, 1-36, doi:10.1007/BF02417081, 1886.

Kim, K. T. and Lee, C. W.:Dynamic analysis of asymmetric bladedrotors supported by anisotropic stator, J. Sound Vibrat., 331, 5224-5246, doi:10.1016/j.jsv.2012.06.014, 2012.

Kim, T., Larsen, T. J., and Yde, A.: Investigation of potential extreme load reduction for a two-bladed upwind turbine with partial pitch, Wind Energy, 18, 1403-1419, doi:10.1002/we.1766, 2015.

Kirchgäßner, B.: ARLIS - A Program System for Aeroelastic Analysis of Rotating Linear Systems, in: Proceedings of the European Wind Energy Conference 1984 edited by: Palz, W., Hamburg, Germany, 253-264, 1984.

Krenk, S.: Non-linear modeling and analysis of solids and structures, Cambridge University Press, Cambridge, 2009.

Larsen, T. J. and Kim, T.: Experimental and Numerical Study of a New Dynamic Phenomenon for Two-bladed Wind Turbines, in: Proceedings of the Twenty-fifth (2015) International Ocean and Polar Engineering Conference, International Society of Offshore and Polar Engineers (ISOPE), Kona, Big Island, Hawaii, USA, 547-553, 2015.

Lazarus, A. and Thomas, O.: A harmonic-based method for computing the stability of periodic solutions of dynamical systems, Comptes Rendus Mécanique, 338, 510-517, doi:10.1016/j.crme.2010.07.020, 2010.

Lee, C. W. and Kim, M. E.: Separation and identification of travelling wave modes in rotating disk via directional spectral analysis, J. Sound Vibrat., 187, 851-864, doi:10.1006/jsvi.1995.0568, 1995.

Lee, C. W., Han, D. J., Suh, J. H., and Hong, S. W.: Modal analysis of periodically time-varying linear rotor systems, J. Sound Vibrat., 303, 553-574, 2007.

Meirovitch, L.: Methods of Analytical Dynamics, McGraw-Hill, New York, 1970.

Riziotis, V. A., Politis, E. S., Voutsinas, S. G., and Chaviaropoulos, P. K.: Stability Analysis of Pitch-regulated, Variable-speed Wind Turbines in Closed Loop Operation Using a Linear Eigenvalue Approach, Wind Energy, 11, 517-535, 2008.

Skjoldan, P. F.: Modal Dynamics of Wind Turbines with Anisotropic Rotors, in: 47th AIAA Aerospace Sciences Meeting Including The New Horizons Forum and Aerospace Exposition, AIAA, Reno, 2009.

Skjoldan, P. F. and Hansen, M. H.: On the similarity of the Coleman and Lyapunov-Floquet transformations for modal analysis of bladed rotor structures, J. Sound Vibrat., 327, 424-439, 2009.

Skjoldan, P. F. and Hansen, M. H.: Effects of extreme wind shear on aeroelastic modal damping of wind turbines, Wind Energy, 16, 401-415, doi:10.1002/we.1495, 2013.

Stol, K., Balas, M., and Bir, G.: Floquet Modal Analysis of a Teetered-Rotor Wind Turbine, J. Sol. Energy Eng., 124, 364 371, 2002.

van Engelen, T. G. and Braam, H.: TURBU Offshore, computer program for frequency-domain analysis of horizontal-axis offshore wind turbines - Implementation, Technical Report ECN-C-04079, ECN, Petten, the Netherlands, 2004. 
van Solingen, E.: Linear individual pitch control design for two-bladed wind turbines, Wind Energy, 18, 677-697, doi:10.1002/we.1720, 2015.

van Solingen, E., Beerens, J., Mulders, S., De Breuker, R., and van Wingerden, J.: Control design for a two-bladed downwind teeterless damped free-yaw wind turbine, Mechatronics, 36, 77-96, doi:10.1016/j.mechatronics.2016.03.008, 2016a.

van Solingen, E., Fleming, P. A., Scholbrock, A., van Wingerden, J. W.: Field testing of linear individual pitch control on the twobladed controls advanced research turbine, Wind Energy, 19, 421-436, doi:10.1002/we.1841, 2016 b.

Wang, N. and Wright, A.: Disturbance Accommodating Control based Independent Blade Pitch Control Design on CART2, 34th Wind Energy Symposium, January 2016, San Diego, CA, USA, 1-17, doi:10.2514/6.2016-1736, 2016.
Warmbrodt, W. and Friedmann, P. P.: Coupled Rotor/Tower Aeroelastic Analysis of Large Horizontal Axis Wind Turbines, AIAA J., 18, 1118-1124, 1980.

Wendell, J. H.: Simplified Aeroelastic Modeling of Horizontal Axis Wind Turbines, MIT Report, Aeroelastic and Structures Research Lab., Cambridge, MA, USA, 1982.

$\mathrm{Xu}, \mathrm{J}$. and Gasch, R.: Modale Behandlung linearer periodisch zeitvarianter Bewegungsgleichungen, Arch. Appl. Mech., 65, 178193, 1995. 